\title{
COMPUTATIONALLY PRIVATE RANDOMIZING POLYNOMIALS AND THEIR APPLICATIONS
}

\author{
Benny Applebaum, Yuval Ishai, and Eyal Kushilevitz
}

\begin{abstract}
Randomizing polynomials allow representing a function $f(x)$ by a low-degree randomized mapping $\hat{f}(x, r)$ whose output distribution on an input $x$ is a randomized encoding of $f(x)$. It is known that any function $f$ in uniform $\oplus \mathrm{L} /$ poly (and in particular in $\mathrm{NC}^{1}$ ) can be efficiently represented by degree-3 randomizing polynomials. Such a degree3 representation gives rise to an $\mathrm{NC}_{4}^{0}$ representation, in which every bit of the output depends on only four bits of the input.

In this paper, we study the relaxed notion of computationally private randomizing polynomials, where the output distribution of $\hat{f}(x, r)$ should only be computationally indistinguishable from a randomized encoding of $f(x)$. We construct degree-3 randomizing polynomials of this type for every polynomial-time computable function, assuming the existence of a cryptographic pseudorandom generator (PRG) in uniform $\oplus \mathrm{L} /$ poly. (The latter assumption is implied by most standard intractability assumptions used in cryptography.) This result is obtained by combining a variant of Yao's garbled circuit technique with previous "informationtheoretic" constructions of randomizing polynomials.

We present several applications of computationally private randomizing polynomials in cryptography. In particular, we relax the sufficient assumptions for parallel constructions of cryptographic primitives, obtain new parallel reductions between primitives, and simplify the design of constant-round protocols for multiparty computation.
\end{abstract}

Keywords. Cryptography, constant depth circuits, $\mathrm{NC}^{0}$, parallel construction, randomizing polynomials, garbled circuit

Subject classification. 94A60, 68P25, 68Q15.

\section{Introduction}

To what extent can one simplify the task of computing a function $f$ by settling for computing some (possibly randomized) encoding of its output? The study of this question was initiated in the context of secure multi-party computation (Ishai \& Kushilevitz 2000, 2002), and has recently found applications to 
parallel constructions of cryptographic primitives (Applebaum et al. 2004). In this paper we consider a relaxed variant of this question and present some new constructions and cryptographic applications.

The above question can be formally captured by the following notion. We say that a function $\hat{f}(x, r)$ is a randomized encoding of a function $f(x)$ if its output distribution depends only on the output of $f$. More precisely, we require that: (1) given $\hat{f}(x, r)$ one can efficiently recover $f(x)$, and (2) given $f(x)$ one can efficiently sample from the distribution of $\hat{f}(x, r)$ induced by a uniform choice of $r$.

This notion of randomized encoding defines a non-trivial relaxation of the usual notion of computing, and thus gives rise to the following question: Can we encode "complex" functions $f$ by "simple" functions $\hat{f}$ ? This question is motivated by the fact that in many cryptographic applications, $\hat{f}$ can be securely used as a substitute for $f$ (Applebaum et al. 2004; Ishai \& Kushilevitz 2000). For instance, if $f$ is a one-way function then so is $\hat{f}$. It should be noted that different applications motivate different interpretations of the term "simple" above. In the context of multi-party computation, one is typically interested in minimizing the algebraic degree of $\hat{f}$, viewing it as a vector of multivariate polynomials over a finite field. In this context, $\hat{f}$ was referred to as a representation of $f$ by randomizing polynomials (Ishai \& Kushilevitz 2000). In other contexts it is natural to view $\hat{f}$ as a function over binary strings and attempt to minimize its parallel time complexity (Applebaum et al. 2004). From here on, we will refer to $\hat{f}$ as a "randomized encoding" of $f$ (or simply "encoding" for short) except when we wish to stress that we are interested in minimizing the degree.

It was shown in Ishai \& Kushilevitz $(2000,2002)$ that every function $f$ in uniform $\oplus \mathrm{L} /$ poly $^{1}$ can be efficiently represented by degree- 3 randomizing polynomials over $\mathrm{GF}(2)$. (This result generalizes to arbitrary finite fields or even rings, allowing efficient degree-3 representations of various counting logspace classes. See Cramer et al. 2003; Ishai \& Kushilevitz 2002.) Moreover, every degree-3 encoding can in turn be converted into an $\mathrm{NC}^{0}$ encoding with locality 4, namely one in which every bit of the output depends on only four bits of the input (Applebaum et al. 2004). A major question left open by the

\footnotetext{
${ }^{1}$ For brevity, all complexity classes are assumed to be polynomial-time uniform by default. In particular, the classes uniform $\oplus \mathrm{L} /$ poly, uniform $\mathrm{L} /$ poly and $\mathrm{NC}^{i}$ are all assumed to be polynomial-time uniform. (See Section 2 for definitions of these classes.) The class uniform $\oplus \mathrm{L} /$ poly contains uniform $\mathrm{L} /$ poly and $\mathrm{NC}^{1}$, and is contained in $\mathrm{NC}^{2}$. In the non-uniform setting $\oplus \mathrm{L} /$ poly also contains NL/poly (Wigderson 1994). However, such an inclusion is not known to hold in the uniform setting.
} 
above results is whether every polynomial-time computable function admits an encoding in $\mathrm{NC}^{0}$.

In this work we consider a relaxed notion of computationally private randomized encodings, where requirement (2) above is relaxed to allow sampling from a distribution which is computationally indistinguishable from $\hat{f}(x, r)$. As it turns out, computationally private encodings are sufficient for most applications. Thus, settling the latter question for the relaxed notion may be viewed as a second-best alternative.

1.1. Overview of results and techniques. We construct a computationally private encoding in $\mathrm{NC}^{0}$ for every polynomial-time computable function, assuming the existence of a "minimal" cryptographic pseudorandom generator (PRG) (Blum \& Micali 1984; Yao 1982), namely one that stretches its seed by just one bit, in uniform $\oplus \mathrm{L} /$ poly. We refer to the latter assumption as the "Easy PRG" (EPRG) assumption. (This assumption can be slightly relaxed, e.g., to also be implied by the existence of a PRG in uniform NL/poly; see Remark 4.16.) We note that EPRG is a very mild assumption. In particular, it is implied by most concrete intractability assumptions commonly used in cryptography, such as ones related to factoring, discrete logarithm, or lattice problems (see Remark 6.6 in the full version of Applebaum et al. 2004). It is also implied by the existence in uniform $\oplus \mathrm{L} /$ poly of a one-way permutation or, using Håstad et al. (1999), of any regular one-way function (OWF); i.e., a OWF $f=\left\{f_{n}\right\}$ that maps the same (polynomial-time computable) number of elements in $\{0,1\}^{n}$ to every element in $\operatorname{Im}\left(f_{n}\right)$. (This is the case, for instance, for any one-to-one OWF.) ${ }^{2}$ The $\mathrm{NC}^{0}$ encoding we obtain under the EPRG assumption has degree 3 and locality 4 . Its size is nearly linear in the circuit size of the encoded function.

We now give a high-level overview of our construction. Recall that we wish to encode a polynomial-time computable function by an $\mathrm{NC}^{0}$ function. To do this we rely on a variant of Yao's garbled circuit technique (Yao 1986). Roughly speaking, Yao's technique allows one to efficiently "encrypt" a boolean circuit in a way that enables computing the output of the circuit but "hides" any other information about the circuit's input. These properties resemble the ones required for randomized encoding. Moreover, the garbled circuit enjoys a certain level of locality (or parallelism) in the sense that gates are encrypted independently of each other. Specifically, each encrypted gate is obtained by applying some cryptographic primitive (typically, a high-stretch PRG or an

\footnotetext{
${ }^{2}$ Using Håstad et al. (1999) or Haitner et al. (2005) this regularity requirement can be relaxed. See Footnote 14 in the full version of Applebaum et al. (2004).
} 
encryption scheme with special properties), on a constant number of (long) random strings and, possibly, a single input bit. However, the overall circuit might not have constant locality (it might not even be computable in NC) as the cryptographic primitive being used in the gates might be sequential in nature. Thus, the bottleneck of the construction is the parallel time complexity of the primitive being used.

Recently, it was shown in Applebaum et al. (2004) (via "information-theoretic" randomized encoding) that under relatively mild assumptions many cryptographic primitives can be computed in $\mathrm{NC}^{0}$. Hence, we can try to plug one of these primitives into the garbled circuit construction in order to obtain an encoding with constant locality. However, a direct use of this approach would require stronger assumptions ${ }^{3}$ than EPRG and result in an $\mathrm{NC}^{0}$ encoding with inferior parameters. Instead, we use the following variant of this approach.

Our construction consists of three steps. The first step is an $\mathrm{NC}^{0}$ implementation of one-time symmetric encryption using a minimal PRG as an oracle. (Such an encryption allows one to encrypt a single message whose length may be polynomially larger than the key. Note that we are only assuming the existence of a minimal PRG, i.e., a PRG that stretches its seed only by one bit. Such a PRG cannot be directly used to encrypt long messages.) The second and main step of the construction relies on a variant of Yao's garbled circuit technique (Yao 1986) to obtain an encoding in $\mathrm{NC}^{0}$ which uses one-time symmetric encryption as an oracle. By combining these two steps we get an encoding that can be computed by an $\mathrm{NC}^{0}$ circuit which uses a minimal PRG as an oracle. Finally, using the EPRG assumption and Applebaum et al. (2004), we apply a final step of "information-theoretic" encoding to obtain an encoding in $\mathrm{NC}^{0}$ with degree 3 and locality 4 .

The above result gives rise to several types of cryptographic applications, discussed below.

1.1.1. Relaxed assumptions for cryptography in $\mathrm{NC}^{0}$. The question of minimizing the parallel time complexity of cryptographic primitives has been the subject of an extensive body of research (see Applebaum et al. 2004; Naor \& Reingold 1999 and references therein). Pushing parallelism to the extreme, it is natural to ask whether one can implement cryptographic primitives in $\mathrm{NC}^{0}$. While it was known that few primitives, including pseudorandom func-

\footnotetext{
${ }^{3}$ Previous presentations of Yao's garbled circuit relied on primitives that seem less likely to allow an $\mathrm{NC}^{0}$ implementation. Specifically, Beaver et al. (1990) and Naor et al. (1999) require linear stretch PRG and Lindell \& Pinkas (2004) requires symmetric encryption that enjoys some additional properties.
} 
tions (Goldreich et al. 1986), cannot even be implemented in $\mathrm{AC}^{0}$ (Linial et al. 1993), no similar negative results were known for other primitives.

Very recently, it was shown in Applebaum et al. (2004) that the existence of most cryptographic primitives in $\mathrm{NC}^{0}$ follows from their existence in higher complexity classes such as uniform $\oplus \mathrm{L} /$ poly, which is typically a very mild assumption. This result was obtained by combining the results on (informationtheoretic) randomized encodings mentioned above with the fact that the security of most cryptographic primitives is inherited by their randomized encoding.

Using our construction of computationally private encodings, we can further relax the sufficient assumptions for cryptographic primitives in $\mathrm{NC}^{0}$. The main observation is that the security of most primitives is also inherited by their computationally private encoding. This is the case even for relatively "sophisticated" primitives such as public-key encryption, digital signatures, (computationally hiding) commitments, and (interactive or non-interactive) zero-knowledge proofs. Thus, given that these primitives at all exist, their existence in $\mathrm{NC}^{0}$ follows from the EPRG assumption, namely from the existence of a PRG in complexity classes such as uniform $\oplus \mathrm{L} /$ poly. (The above condition is redundant in the case of signatures and commitments, whose existence follows from the existence of a PRG. In Section 1.1.2 we will describe a stronger result for such primitives.) Previously (using Applebaum et al. 2004), the existence of each of these primitives in $\mathrm{NC}^{0}$ would only follow from the assumption that this particular primitive can be implemented in the above classes, a seemingly stronger assumption than EPRG.

It should be noted that we cannot obtain a similar result for some other primitives, such as one-way permutations and collision-resistant hash functions. The results for these primitives obtained in Applebaum et al. (2004) rely on certain regularity properties of the encoding that are lost in the transition to computational privacy.

1.1.2. Parallel reductions between cryptographic primitives. The results of Applebaum et al. (2004) also give rise to new $\mathrm{NC}^{0}$ reductions between cryptographic primitives. (Unlike the results discussed in Section 1.1.1 above, here we consider unconditional reductions that do not rely on unproven assumptions.) In particular, known $\mathrm{NC}^{1}$ reductions from PRG to one-way permutations (Goldreich \& Levin 1989) or even to more general types of one-way functions (Haitner et al. 2005; Håstad et al. 1999; Viola 2005) can be encoded into $\mathrm{NC}^{0}$ reductions (see Remark 6.7 in the full version of Applebaum et al. 2004). However, these $\mathrm{NC}^{0}$ reductions crucially rely on the very simple structure of the $\mathrm{NC}^{1}$ reductions from which they are derived. In particular, it is not 
possible to use the results of Applebaum et al. (2004) for encoding general $\mathrm{NC}^{1}$ reductions (let alone polynomial-time reductions) into $\mathrm{NC}^{0}$ reductions.

As a surprising application of our technique, we get a general "compiler" that converts an arbitrary (polynomial-time) reduction from a primitive $\mathcal{P}$ to a PRG into an $\mathrm{NC}^{0}$ reduction from $\mathcal{P}$ to a PRG. This applies to all primitives $\mathcal{P}$ that are known to be equivalent to a one-way function, and whose security is inherited by their computationally private encoding. In particular, we conclude that symmetric encryption, commitment, and digital signatures are all $\mathrm{NC}^{0}$ reducible to a minimal $\mathrm{PRG}$ and hence also to a one-way permutation or more general types of one-way functions. (By symmetric encryption we refer to probabilistic stateless encryption for multiple messages, where the parties do not maintain any state information other than the key. If parties are allowed to maintain synchronized states, symmetric encryption can be easily reduced in $\mathrm{NC}^{0}$ to a PRG.)

No parallel reductions of this type were previously known, even in NC. The known construction of commitment from a PRG (Naor 1991) requires a linearstretch PRG (expanding $n$ bits into $n+\Omega(n)$ bits), which is not known to be reducible in parallel to a minimal PRG. Other primitives, such as symmetric encryption and signatures, were not even known to be reducible in parallel to a polynomial-stretch PRG. For instance, the only previous parallel construction of symmetric encryption from a "low-level" primitive is based on the parallel PRF construction of Naor \& Reingold (1999). This yields an $\mathrm{NC}^{1}$ reduction from symmetric encryption to synthesizers, a stronger primitive than a PRG. Thus, we obtain better parallelism and at the same time rely on a weaker primitive. The price we pay is that we cannot generally guarantee parallel decryption. (See Section 5.2 for further discussion.)

An interesting feature of the new reductions is their non-black-box use of the underlying PRG. That is, the "code" of the $\mathrm{NC}^{0}$ reduction we get (implementing $\mathcal{P}$ using an oracle to a PRG) depends on the code of the PRG. This should be contrasted with most known reductions in cryptography, which make a black-box use of the underlying primitive. In particular, this is the case for the above-mentioned $\mathrm{NC}^{0}$ reductions based on Applebaum et al. (2004). (See Reingold et al. 2004 for a thorough taxonomy of reductions in cryptography.)

1.1.3. Application to secure computation. The notion of randomizing polynomials was originally motivated by the goal of minimizing the round complexity of secure multi-party computation (Ben-Or et al. 1988; Chaum et al. 1988; Goldreich et al. 1987; Yao 1986). The main relevant observations made in Ishai \& Kushilevitz (2000) were that: (1) the round complexity of most gen- 
eral protocols from the literature is related to the degree of the function being computed; and (2) if $f$ is represented by a vector $\hat{f}$ of degree- $d$ randomizing polynomials, then the task of securely computing $f$ can be reduced to that of securely computing some deterministic degree- $d$ function $\hat{f}^{\prime}$ which is closely related to $\hat{f}$. This reduction from $f$ to $\hat{f}^{\prime}$ is fully non-interactive, in the sense that a protocol for $f$ can be obtained by invoking a protocol for $\hat{f}$ and applying a local computation on its outputs (without additional interaction).

A useful corollary of our results is that under the EPRG assumption, the task of securely computing an arbitrary polynomial-time computable function $f$ reduces (non-interactively) to that of securely computing a related degree-3 function $\hat{f}^{\prime}$. This reduction is only computationally secure. Thus, even if the underlying protocol for $\hat{f}^{\prime}$ is secure in an information-theoretic sense, the resulting protocol for $f$ will only be computationally secure. (In contrast, previous constructions of randomizing polynomials maintained information-theoretic security, but only efficiently applied to restricted function classes such as uniform $\oplus \mathrm{L} /$ poly.) This reduction gives rise to new, conceptually simpler, constantround protocols for general functions. For instance, a combination of our result with the classical "BGW protocol" (Ben-Or et al. 1988) gives a simpler, and in some cases more efficient, alternative to the constant-round protocol of Beaver, Micali \& Rogaway (1990) (though relies on a stronger assumption).

Organization. Following some preliminaries (Section 2), in Section 3 we review previous notions of randomized encoding and define our new notion of computationally private encoding. In Section 4 we construct a computationally private encoding in $\mathrm{NC}^{0}$ for every polynomial-time computable function. Applications of this construction are discussed in Section 5. Finally, the proof of the privacy of the garbled circuit construction is deferred to Section 6 .

\section{Preliminaries}

Probability notation. We let $U_{n}$ denote a random variable uniformly distributed over $\{0,1\}^{n}$. If $X$ is a probability distribution, or a random variable, we write $x \leftarrow X$ to indicate that $x$ is a sample taken from $X$. The statistical distance between discrete probability distributions $Y$ and $Y^{\prime}$, denoted $\mathrm{SD}\left(Y, Y^{\prime}\right)$, is defined as the maximum, over all functions $A$, of the distinguishing advantage $\left|\operatorname{Pr}[A(Y)=1]-\operatorname{Pr}\left[A\left(Y^{\prime}\right)=1\right]\right|$. A function $\varepsilon(\cdot)$ is said to be negligible if $\varepsilon(n)<n^{-c}$ for any constant $c>0$ and sufficiently large $n$. For two distribution ensembles $\left\{X_{n}\right\}_{n \in \mathbb{N}}$ and $\left\{Y_{n}\right\}_{n \in \mathbb{N}}$, we write $\left\{X_{n}\right\}_{n \in \mathbb{N}} \equiv\left\{Y_{n}\right\}_{n \in \mathbb{N}}$ if $X_{n}$ and $Y_{n}$ are identically distributed, and say that the two ensembles are 
statistically indistinguishable if $\operatorname{SD}\left(X_{n}, Y_{n}\right)$ is negligible in $n$. A weaker notion of closeness between distributions is that of computational indistinguishability: We write $\left\{X_{n}\right\}_{n \in \mathbb{N}} \stackrel{c}{=}\left\{Y_{n}\right\}_{n \in \mathbb{N}}$ if for every (non-uniform) polynomial-size circuit family $\left\{A_{n}\right\}$, the distinguishing advantage $\left|\operatorname{Pr}\left[A_{n}\left(X_{n}\right)=1\right]-\operatorname{Pr}\left[A_{n}\left(Y_{n}\right)=1\right]\right|$ is negligible. (We will sometimes simplify notation and write $X_{n} \stackrel{c}{\equiv} Y_{n}$.) By default we adapt this non-uniform notion of indistinguishability. However, our results also apply in a uniform setting in which adversaries are probabilistic polynomial-time algorithms.

We will rely on several standard facts about computational indistinguishability (cf. Goldreich 2001, Chapter 2).

FACT 2.1. For every distribution ensembles $X, Y$ and $Z$, if $X \stackrel{c}{\equiv} Y$ and $Y \stackrel{c}{\equiv} Z$ then $X \stackrel{c}{\equiv} Z$.

That is, computational indistinguishability is transitive. The following fact asserts that computational indistinguishability is preserved under multiple independent samples:

FACT 2.2. Let $\left\{X_{n}\right\},\left\{X_{n}^{\prime}\right\},\left\{Y_{n}\right\}$ and $\left\{Y_{n}^{\prime}\right\}$ be distribution ensembles. Suppose that $X_{n} \stackrel{c}{\equiv} Y_{n}$ and $X_{n}^{\prime} \stackrel{c}{\equiv} Y_{n}^{\prime}$. Then $\left(X_{n} \times X_{n}^{\prime}\right) \stackrel{c}{\equiv}\left(Y_{n} \times Y_{n}^{\prime}\right)$, where $A \times B$ denotes the product distribution of $A, B$ (i.e., the joint distribution of independent samples from $A$ and $B$ ).

Another basic fact is that computational indistinguishability is preserved under efficient computation:

FACT 2.3. Suppose that the distribution ensembles $\left\{X_{n}\right\}$ and $\left\{Y_{n}\right\}$ are computationally indistinguishable. Then for every polynomial-time computable function $f$ we have $f\left(X_{n}\right) \stackrel{c}{\equiv} f\left(Y_{n}\right)$.

Consider a case in which two probabilistic (possibly computationally unbounded) algorithms behave "similarly" on every input, in the sense that their output distributions are computationally indistinguishable. The following two facts deal with such a situation. Fact 2.4 asserts that an efficient procedure that gets an oracle access to one of these algorithms cannot tell which algorithm it communicates with. Fact 2.5 asserts that the outputs of these algorithms cannot be distinguished with respect to any (not necessarily efficiently samplable) input distribution. These facts will allow us to argue that, in most applications, computationally private encodings can be securely used as substitutes for perfectly private encodings. 
FACT 2.4. Let $X$ and $Y$ be probabilistic algorithms such that $X\left(z_{n}\right) \stackrel{c}{\equiv} Y\left(z_{n}\right)$ for every string family $\left\{z_{n}\right\}$ where $z_{n} \in\{0,1\}^{n}$. Then, for any (non-uniform) polynomial-time oracle machine $A$, we have $A^{X}\left(1^{n}\right) \stackrel{c}{\equiv} A^{Y}\left(1^{n}\right)$ (where $A$ does not have access to the random coins of the given probabilistic oracle).

FACT 2.5. Let $X$ and $Y$ be probabilistic algorithms such that $X\left(z_{n}\right) \stackrel{c}{\equiv} Y\left(z_{n}\right)$ for every string family $\left\{z_{n}\right\}$ where $z_{n} \in\{0,1\}^{n}$. Then, for every distribution ensemble $\left\{Z_{n}\right\}$ where $Z_{n}$ is distributed over $\{0,1\}^{n}$, we have $\left(Z_{n}, X\left(Z_{n}\right)\right) \stackrel{c}{\equiv}$ $\left(Z_{n}, Y\left(Z_{n}\right)\right)$.

Circuits. Boolean circuits are defined in a standard way. That is, we define a boolean circuit $C$ as a directed acyclic graph with labeled, ordered vertices of the following types: (1) input vertices, each labeled with a literal $x_{i}$ or $\bar{x}_{i}$ and having fan-in 0; (2) gate vertices, labeled with one of the boolean functions AND, OR and having fan-in 2; (3) output vertices, labeled "output" and having fan-in 1 and fan-out 0 . The edges of the circuit are referred to as wires. A wire that outgoes from an input vertex is called an input wire, and a wire that enters an output vertex is called an output wire. Any input $x \in\{0,1\}^{n}$ assigns a unique value to each wire in the natural way. The output value of $C$, denoted $C(x)$, contains the values of the output wires according to the given predefined order. The size of a circuit, denoted $|C|$, is the number of wires in $C$, and its depth is the maximum distance from an input to an output (i.e. the length of the longest directed path in the graph).

$\mathrm{NC}^{i}$ reductions. A circuit with an oracle access to a function $g:\{0,1\}^{*} \rightarrow$ $\{0,1\}^{*}$ is a circuit that contains, in addition to the bounded fan-in OR, AND gates, special oracle gates with unbounded fan-in that compute the function $g$. We say that $f:\{0,1\}^{*} \rightarrow\{0,1\}^{*}$ is $\mathrm{NC}^{i}$ reducible to $g$, and write $f \in \mathrm{NC}^{i}[g]$, if $f$ can be computed by a uniform family of polynomial size, $O\left(\log ^{i} n\right)$ depth circuits with oracle gates to $g$. (Oracle gates are treated just as AND/OR gates when defining depth.) Note that if $f \in \mathrm{NC}^{i}[g]$ and $g \in \mathrm{NC}^{j}$ then $f \in \mathrm{NC}^{i+j}$.

Locality and degree. We say that $f$ is $c$-local if each of its output bits depends on at most $c$ input bits. For a constant $c$, the class non-uniform $\mathrm{NC}_{c}^{0}$ includes all $c$-local functions. We will sometimes view the binary alphabet as the finite field $\mathcal{F}=\mathrm{GF}(2)$, and say that a function $f$ has degree $d$ if each of its output bits can be expressed as a multivariate polynomial of degree (at most) $d$ in the input bits. 
Complexity classes. For brevity, we use the (somewhat non-standard) convention that all complexity classes are polynomial-time uniform by default. For instance, $\mathrm{NC}^{0}$ refers to the class of functions admitting polynomial-time uniform $\mathrm{NC}^{0}$ circuits, whereas non-uniform $\mathrm{NC}^{0}$ refers to the class of functions admitting non-uniform $\mathrm{NC}^{0}$ circuits. We let uniform NL/poly (resp., uniform $\oplus \mathrm{L} /$ poly) denote the class of boolean functions computed by NL (resp., $\oplus \mathrm{L}$ ) Turing machines taking a polynomial-time uniform advice. (The class uniform $\oplus \mathrm{L} /$ poly contains the classes uniform $\mathrm{L} /$ poly and $\mathrm{NC}^{1}$ and is contained in $\mathrm{NC}^{2}$.) We extend boolean complexity classes, such as uniform NL/poly and uniform $\oplus \mathrm{L} /$ poly, to include non-boolean functions by letting the representation include $\ell(n) \log$-space Turing machines, one for each output bit, taking the same uniform advice. Similarly, we denote by P (resp. BPP) the class of functions that can be computed in polynomial time (resp. probabilistic polynomial time). For instance, a function $f:\{0,1\}^{n} \rightarrow\{0,1\}^{\ell(n)}$ is in BPP if there exists a probabilistic polynomial-time machine $A$ such that $\operatorname{Pr}[A(x) \neq f(x)] \leq 2^{-n}$ for every $x \in\{0,1\}^{n}$, where the probability is taken over the internal coin tosses of $A$.

\section{Randomized encodings}

We now review the notions of randomized encoding and randomizing polynomials from Applebaum et al. (2004) and Ishai \& Kushilevitz (2000, 2002), and introduce the new computationally private variant discussed in this paper. The following definition is from Applebaum et al. (2004).

Definition 3.1. Let $f:\{0,1\}^{n} \rightarrow\{0,1\}^{\ell}$ be a function. We say that a function $\hat{f}:\{0,1\}^{n} \times\{0,1\}^{m} \rightarrow\{0,1\}^{s}$ is a $\delta$-correct, $\varepsilon$-private randomized encoding of $f$ if it satisfies the following:

- $\delta$-correctness: There exists an algorithm $B$, called a decoder, such that for any input $x \in\{0,1\}^{n}, \operatorname{Pr}\left[B\left(\hat{f}\left(x, U_{m}\right)\right) \neq f(x)\right] \leq \delta$.

- $\varepsilon$-privacy: There exists a randomized algorithm $S$, called a simulator, such that for any $x \in\{0,1\}^{n}, \mathrm{SD}\left(S(f(x)), \hat{f}\left(x, U_{m}\right)\right) \leq \varepsilon$.

We refer to the second input of $\hat{f}$ as its random input, and to $m, s$ as the randomness complexity and the output complexity of $\hat{f}$ respectively. The complexity of $\hat{f}$ is defined to be $m+s$.

We say that $\hat{f}$ is a representation (or encoding) of $f$ by degree- $d$ randomizing polynomials if each of its output bits can be computed by a multivariate polynomial over $\operatorname{GF}(2)$ of degree at most $d$ in the inputs. 
Definition 3.1 naturally extends to infinite functions $f:\{0,1\}^{*} \rightarrow\{0,1\}^{*}$. In this case, the parameters $\ell, m, s, \delta, \varepsilon$ are all viewed as functions of the input length $n$, and the algorithms $B, S$ receive $1^{n}$ as an additional input. By default, we require $\hat{f}$ to be computable in $\operatorname{poly}(n)$ time whenever $f$ is. In particular, both $m(n)$ and $s(n)$ are polynomially bounded. We also require both the decoder and the simulator algorithms to be efficient.

Several variants of randomized encodings were considered in Applebaum et al. (2004). Correctness (resp., privacy) is said to be perfect when $\delta=0$ (resp. $\varepsilon=0$ ) or statistical when $\delta(n)$ (resp. $\varepsilon(n)$ ) is negligible. In order to preserve the security of some primitives (such as pseudorandom generators or one-way permutations) even perfect correctness and privacy might not suffice and additional requirements should be introduced. An encoding is said to be balanced if it admits a perfectly private simulator $S$ such that $S\left(U_{\ell}\right) \equiv U_{s}$. It is said to be stretch preserving if $s=\ell+m$. We say that $\hat{f}$ is a statistical randomized encoding of $f$ if it is both statistically correct and statistically private, and that it is a perfect randomized encoding if it is perfectly correct and private, balanced, and stretch preserving. In this work, we abandon the information-theoretic setting and relax the privacy requirement to be computational. That is, we require the ensembles $S\left(1^{n}, f_{n}(x)\right)$ and $\hat{f}_{n}\left(x, U_{m(n)}\right)$ to be computationally indistinguishable.

Definition 3.2. Let $f=\left\{f_{n}:\{0,1\}^{n} \rightarrow\{0,1\}^{\ell(n)}\right\}_{n \in \mathbb{N}}$ be a function family. We say that the function family $\hat{f}=\left\{\hat{f}_{n}:\{0,1\}^{n} \times\{0,1\}^{m(n)} \rightarrow\{0,1\}^{s(n)}\right\}_{n \in \mathbb{N}}$ is a computational randomized encoding of $f$ (or computational encoding for short) if it satisfies the following requirements:

- Statistical correctness: There exists a polynomial-time decoder $B$ such that for any $n$ and any input $x \in\{0,1\}^{n}, \operatorname{Pr}\left[B\left(1^{n}, \hat{f}_{n}\left(x, U_{m(n)}\right)\right) \neq\right.$ $\left.f_{n}(x)\right] \leq \delta(n)$ for some negligible function $\delta(n)$.

- Computational privacy: There exists a probabilistic polynomial-time simulator $S$ such that for any family of strings $\left\{x_{n}\right\}_{n \in \mathbb{N}}$ where $\left|x_{n}\right|=n$, we have $S\left(1^{n}, f_{n}\left(x_{n}\right)\right) \stackrel{\text { c }}{\equiv} \hat{f}_{n}\left(x_{n}, U_{m(n)}\right)$.

We will also refer to perfectly correct computational encodings, where the statistical correctness requirement is strengthened to perfect correctness. In fact, our main construction yields a perfectly correct encoding.

REMARK 3.3. The above definition uses $n$ both as an input length parameter and as a cryptographic "security parameter" quantifying computational privacy. When describing our construction, it will be convenient to use a separate 
parameter $k$ for the latter, where computational privacy will be guaranteed as long as $k \geq n^{\epsilon}$ for some constant $\epsilon>0$.

The function classes $\mathcal{S} \mathcal{R E N}$ and $\mathcal{P} \mathcal{R} \mathcal{E N}$ were introduced in Applebaum et al. (2004) to capture the power of statistical and perfect randomized encodings in $\mathrm{NC}^{0}$. We define a similar class $\mathcal{C} \mathcal{R} \mathcal{E}$.

Definition 3.4. The class $\mathcal{C R \mathcal { E N }}$ (resp., $\mathcal{S} \mathcal{R} \mathcal{E N}, \mathcal{P} \mathcal{R} \mathcal{E} \mathcal{N}$ ) is the class of functions $f$ admitting a computational (resp., statistical, perfect) randomized encoding $\hat{f}$ in $\mathrm{NC}^{0}$. (As usual, $\mathrm{NC}^{0}$ is polynomial-time uniform.)

It follows from the definitions that $\mathcal{P} \mathcal{R E N} \subseteq \mathcal{S} \mathcal{R} \mathcal{E} \mathcal{N} \subseteq \mathcal{C R E \mathcal { N }}$. Moreover, it is known that uniform $\oplus \mathrm{L} /$ poly $\subseteq \mathcal{P} \mathcal{R E \mathcal { N }}$ and uniform NL/poly $\subseteq$ $\mathcal{S} \mathcal{R} \mathcal{E} \mathcal{N}$ (Applebaum et al. 2004). (We cannot use the fact that NL/poly $\subseteq$ $\oplus \mathrm{L} /$ poly to conclude that uniform NL/poly $\subseteq \mathcal{P} \mathcal{R} \mathcal{E} \mathcal{N}$, since this inclusion is only known to hold in the non-uniform setting.)

We end this section by considering the following intuitive composition property: Suppose we encode $f$ by $g$, and then view $g$ as a single-argument function and encode it again. Then the resulting function (parsed appropriately) is an encoding of $f$. The following lemma was stated in Applebaum et al. (2004) for the statistical and perfect variants of randomized encodings; we extend it here to the computational variant.

LEMma 3.5 (Composition). Let $g\left(x, r_{g}\right)$ be a computational encoding of $f(x)$ and $h\left(\left(x, r_{g}\right), r_{h}\right)$ a computational encoding of $g\left(\left(x, r_{g}\right)\right)$, viewing the latter as a single-argument function. Then the function $\hat{f}\left(x,\left(r_{g}, r_{h}\right)\right) \stackrel{\text { def }}{=} h\left(\left(x, r_{g}\right), r_{h}\right)$ is a computational encoding of $f(x)$ whose random inputs are $\left(r_{g}, r_{h}\right)$. Moreover, if $g, h$ are perfectly correct then so is $\hat{f}$.

Proof. We start with correctness. Let $B_{g}$ be a $\delta_{g}(n)$-correct decoder for $g$ and $B_{h}$ a $\delta_{h}\left(n+m_{g}(n)\right)$-correct decoder for $h$, where $m_{g}(n)$ is the randomness complexity of $g$. Define a decoder $B$ for $\hat{f}$ by $B(\hat{y})=B_{g}\left(B_{h}(\hat{y})\right)$. The decoder $B$ errs only if either $B_{h}$ or $B_{g}$ err. Thus, by the union bound we have for every $x \in\{0,1\}^{n}$,

$$
\begin{aligned}
\operatorname{Pr}_{r_{g}, r_{h}}\left[B\left(1^{n}, \hat{f}\left(x,\left(r_{g}, r_{h}\right)\right) \neq f(x)\right] \leq\right. & \operatorname{Pr}_{r_{g}, r_{h}}\left[B_{h}\left(1^{n}, h\left(\left(x, r_{g}\right), r_{h}\right)\right) \neq g\left(x, r_{g}\right)\right] \\
& +\operatorname{Pr}_{r_{g}}\left[B_{g}\left(1^{n}, g\left(x, r_{g}\right)\right) \neq f(x)\right] \\
\leq & \delta_{h}\left(n+m_{g}(n)\right)+\delta_{g}(n),
\end{aligned}
$$


and so $B$ is perfectly correct if both the decoders of $h$ and $g$ are perfectly correct. Moreover, since $m_{g}(n)$ is polynomial in $n$, if the decoders of $h$ and $g$ are statistically correct then so is $B$.

To prove computational privacy, we again compose the computationally private simulators of $g$ and $h$, this time in an opposite order. Specifically, let $S_{g}$ be a computationally private simulator for $g$ and $S_{h}$ be computationally private simulator for $h$. We define a simulator $S$ for $\hat{f}$ by $S(y)=S_{h}\left(S_{g}(y)\right)$. Letting $m_{g}(n)$ and $m_{h}(n)$ denote the randomness complexity of $g$ and $h$, respectively, and $\left\{x_{n}\right\}_{n \in \mathbb{N}}$ be a family of strings where $\left|x_{n}\right|=n$, we have

$$
\begin{aligned}
S_{h}\left(S_{g}\left(f\left(x_{n}\right)\right)\right) & \stackrel{c}{\equiv} S_{h}\left(g\left(x_{n}, U_{m_{g}(n)}\right)\right) & & \text { (comp. privacy of } g \text {, Fact 2.3) } \\
& \stackrel{c}{\equiv} h\left(\left(x_{n}, U_{m_{g}(n)}\right), U_{m_{h}(n)}\right) & & \text { (comp. privacy of } h \text {, Fact 2.5). }
\end{aligned}
$$

Hence, the transitivity of the relation $\stackrel{c}{\equiv}$ (Fact 2.1 ) finishes the proof.

It follows as a special case that the composition of a computational encoding with a perfect or statistical encoding is a computational encoding.

REMARK 3.6. It is known that any $f \in \mathcal{P} \mathcal{R E \mathcal { N }}$ (resp., $f \in \mathcal{S} \mathcal{R E N}$ ) admits a perfect (resp., statistical) encoding of degree 3 and locality 4 (Applebaum et al. 2004). The same holds for the class $\mathcal{C} \mathcal{R E N}$, since we can encode a function $f \in \mathcal{C} \mathcal{R} \mathcal{E}$ by a computational encoding $g$ in $\mathrm{NC}^{0}$ and then encode $g$ using a perfect encoding $h$ of degree 3 and locality 4 (promised by the fact that $\left.\mathrm{NC}^{0} \subseteq \mathcal{P} \mathcal{R} \mathcal{E} \mathcal{N}\right)$. By Lemma 3.5, the function $h$ is a computational encoding for $f$ of degree 3 and locality 4 . Moreover, the complexity of $h$ is linearly related to the complexity of $g$.

\section{Computational encoding in $\mathrm{NC}^{0}$ for efficiently computable functions}

In this section we construct a perfectly correct computational encoding of degree 3 and locality 4 for every efficiently computable function. Our construction consists of three steps. In Section 4.1, we describe an $\mathrm{NC}^{0}$ implementation of one-time symmetric encryption using a minimal PRG as an oracle (i.e., a PRG that stretches its seed by just one bit). In Section 4.2 we describe the main step of the construction, in which we encode an arbitrary circuit using an $\mathrm{NC}^{0}$ circuit which uses one-time symmetric encryption as an oracle. This step is based on a variant of Yao's garbled circuit technique (Yao 1986). Combining the first two steps, we get a computational encoding in $\mathrm{NC}^{0}$ with an oracle to a minimal PRG. Finally, in Section 4.3, we derive the main result by relying on the existence of an "easy PRG", namely, a minimal PRG in uniform $\oplus \mathrm{L} /$ poly. 
4.1. From PRG to one-time encryption. An important tool in our construction is a one-time symmetric encryption; that is, a (probabilistic) privatekey encryption that is semantically secure (Goldwasser \& Micali 1984) for encrypting a single message. We describe an $\mathrm{NC}^{0}$ reduction from such an encryption to a minimal PRG, stretching its seed by a single bit. We start by defining minimal PRG and one-time symmetric encryption.

Definition 4.1. A pseudorandom generator (PRG) is a deterministic polynomial-time algorithm, $G$, satisfying the following two conditions:

- Expansion: There exists a function $\ell(k): \mathbb{N} \rightarrow \mathbb{N}$ satisfying $\ell(k)>k$ for all $k \in \mathbb{N}$, such that $|G(x)|=\ell(|x|)$ for all $x \in\{0,1\}^{*}$.

- Pseudorandomness: The ensembles $\left\{G\left(U_{k}\right)\right\}_{k \in \mathbb{N}}$ and $\left\{U_{\ell(k)}\right\}_{k \in \mathbb{N}}$ are computationally indistinguishable.

A PRG that stretches its input by one bit (i.e., $\ell(k)=k+1$ ) is referred to as a minimal PRG. When $\ell(k)=k+\Omega(k)$ we say that $G$ is a linear-stretch PRG. We refer to $G$ as a polynomial-stretch PRG if $\ell(k)=\Omega\left(k^{c}\right)$ for some constant $c>1$.

Definition 4.2. A one-time symmetric encryption scheme is a pair $(E, D)$ of probabilistic polynomial-time algorithms satisfying the following conditions:

- Correctness: For every $k$-bit key $e$ and for every plaintext $m \in\{0,1\}^{*}$, the algorithms $E, D$ satisfy $D_{e}\left(E_{e}(m)\right)=m$ (where $E_{e}(m) \stackrel{\text { def }}{=} E(e, m)$ and similarly for $D$ ).

- Security: For every polynomial $\ell(\cdot)$, and any families of plaintexts $\left\{x_{k}\right\}_{k \in \mathbb{N}}$ and $\left\{x_{k}^{\prime}\right\}_{k \in \mathbb{N}}$ where $x_{k}, x_{k}^{\prime} \in\{0,1\}^{\ell(k)}$, we have

$$
\left\{E_{U_{k}}\left(x_{k}\right)\right\}_{k \in \mathbb{N}} \stackrel{c}{\equiv}\left\{E_{U_{k}}\left(x_{k}^{\prime}\right)\right\}_{k \in \mathbb{N}} .
$$

The integer $k$ serves as the security parameter of the scheme. The scheme is said to be an $\ell(\cdot)$-one-time symmetric encryption scheme if correctness and security hold with respect to plaintexts whose length is bounded by $\ell(k)$.

The above definition enables one to securely encrypt polynomially long messages under short keys. This is an important feature that will be used in our garbled circuit construction described in Section 4.2. In fact, it would 
suffice for our purposes to encrypt messages of some fixed polynomial ${ }^{4}$ length, say $\ell(k)=k^{2}$. This could be easily done in $\mathrm{NC}^{0}$ if we had oracle access to a PRG with a corresponding stretch. Given such a PRG $G$, the encryption can be defined by $E_{e}(m)=G(e) \oplus m$ and the decryption by $D_{e}(c)=G(e) \oplus c$. However, we would like to base our construction on a PRG with a minimal stretch.

From the traditional "sequential" point of view, such a minimal PRG is equivalent to a PRG with an arbitrary polynomial stretch (cf. Goldreich 2001, Thm. 3.3.3). In contrast, this is not known to be the case with respect to parallel reductions. It is not even known whether a linear-stretch PRG is NC-reducible to a minimal PRG (see Viola 2005 for some relevant negative results). Thus, a minimal PRG is a more conservative assumption from the point of view of parallel cryptography. Moreover, unlike a PRG with linear stretch, a minimal PRG is reducible in parallel to one-way permutations and other types of one-way functions (Applebaum et al. 2004; Håstad et al. 1999; Viola 2005).

The above discussion motivates a direct parallel construction of one-time symmetric encryption using a minimal PRG, i.e., a construction that does not rely on a "stronger" type of PRG as an intermediate step. We present such an $\mathrm{NC}^{0}$ construction below.

Construction 4.3 (From PRG to one-time symmetric encryption). Let $G$ be a minimal PRG that stretches its input by a single bit, let e be a $k$-bit key, and let $m$ be a $(k+\ell)$-bit plaintext. Define the probabilistic encryption algorithm

$$
\begin{aligned}
E_{e}\left(m,\left(r_{1}, \ldots, r_{\ell-1}\right)\right) & \stackrel{\text { def }}{=}\left(G(e) \oplus r_{1}, G\left(r_{1}\right) \oplus r_{2}, \ldots, G\left(r_{\ell-2}\right) \oplus r_{l-1}, G\left(r_{\ell-1}\right) \oplus m\right),
\end{aligned}
$$

where $r_{i} \leftarrow U_{k+i}$ serve as the coin tosses of $E$. The decryption algorithm $D_{e}\left(c_{1}, \ldots, c_{\ell-1}\right)$ sets $r_{0}=e, r_{i}=c_{i} \oplus G\left(r_{i-1}\right)$ for $i=1, \ldots, \ell$, and outputs $r_{\ell}$.

We prove the security of Construction 4.3 via a standard hybrid argument.

Lemma 4.4. The scheme $(E, D)$ described in Construction 4.3 is a one-time symmetric encryption scheme.

\footnotetext{
${ }^{4}$ Applying the construction to circuits with a bounded fan-out, even linear length would suffice.
} 
Proof. Construction 4.3 can be easily verified to satisfy the correctness requirement. We now prove the security of this scheme. Assume, towards a contradiction, that Construction 4.3 is not secure. It follows that there is a polynomial $\ell(\cdot)$ and two families of strings $x=\left\{x_{k}\right\}$ and $y=\left\{y_{k}\right\}$, where $\left|x_{k}\right|=\left|y_{k}\right|=k+\ell(k)$, such that the distribution ensembles $E_{e}\left(x_{k}\right)$ and $E_{e}\left(y_{k}\right)$, where $e \leftarrow U_{k}$, can be distinguished by a polynomial size circuit family $\left\{A_{k}\right\}$ with non-negligible advantage $\varepsilon(k)$.

We use a hybrid argument to derive a contradiction. Fix some $k$. For a string $m$ of length $k+\ell(k)$ we define for $0 \leq i \leq \ell(k)$ the distributions $H_{i}(m)$ in the following way. The distribution $H_{0}(m)$ is defined to be $E_{r_{0}}\left(m,\left(r_{1}, \ldots, r_{l-1}\right)\right)$ where $r_{i} \leftarrow U_{k+i}$. For $1 \leq i \leq \ell(k)$, the distribution $H_{i}(m)$ is defined exactly as $H_{i-1}(m)$ only that the string $G\left(r_{i-1}\right)$ is replaced with a random string $w_{i-1}$, which is one bit longer than $r_{i-1}$ (that is, $w_{i-1} \leftarrow U_{k+i}$ ). Observe that for every $m \in\{0,1\}^{k+\ell(k)}$, all the $\ell(k)$ strings of the hybrid $H_{\ell(k)}(m)$ are distributed uniformly and independently (each of them is the result of XOR with a fresh random string $\left.w_{i}\right)$. Therefore, in particular, $H_{\ell(k)}\left(x_{k}\right) \equiv H_{\ell(k)}\left(y_{k}\right)$. Since $H_{0}\left(x_{k}\right) \equiv E_{e}\left(x_{k}\right)$ as well as $H_{0}\left(y_{k}\right) \equiv E_{e}\left(y_{k}\right)$, it follows that our distinguisher $A_{k}$ distinguishes, without loss of generality, between $H_{\ell(k)}\left(x_{k}\right)$ and $H_{0}\left(x_{k}\right)$ with at least $\varepsilon(k) / 2$ advantage. Then, since there are $\ell(k)$ hybrids, there must be $1 \leq i \leq \ell(k)$ such that the neighboring hybrids, $H_{i-1}\left(x_{k}\right), H_{i}\left(x_{k}\right)$, can be distinguished by $A_{k}$ with $\varepsilon(k) / 2 \ell(k)$ advantage.

We now show how to use $A_{k}$ to distinguish a randomly chosen string from an output of the pseudorandom generator. Given a string $z$ of length $k+i$ (that is, either sampled from $G\left(U_{k+i-1}\right)$ or from $\left.U_{k+i}\right)$, we uniformly choose the strings $r_{j} \in\{0,1\}^{k+j}$ for $j=1, \ldots, \ell(k)-1$. We feed $A_{k}$ with the sample $\left(r_{1}, \ldots, r_{i-1}, z \oplus r_{i}, G\left(r_{i}\right) \oplus r_{i+1}, \ldots, G\left(r_{\ell(k)-1}\right) \oplus x_{k}\right)$. If $z$ is a uniformly chosen string then the above distribution is equivalent to $H_{i}\left(x_{k}\right)$. On the other hand, if $z$ is drawn from $G\left(U_{i}\right)$ then the result is distributed exactly as $H_{i-1}\left(x_{k}\right)$, since each of the first $i-1$ entries of $H_{i-1}\left(x_{k}\right)$ is distributed uniformly and independently of the remaining entries (each of these entries was XOR-ed with a fresh and unique random $w_{j}$ ). Hence, we constructed an adversary that breaks the PRG with non-negligible advantage $\varepsilon(k) / 2 \ell(k)$, in contradiction to the hypothesis.

Since the encryption algorithm described in Construction 4.3 is indeed an $\mathrm{NC}^{0}$ circuit with oracle access to a minimal PRG, we get the following lemma.

Lemma 4.5. Let $G$ be a minimal PRG. Then there exists a one-time symmetric encryption scheme $(E, D)$ in which the encryption function $E$ is in $\mathrm{NC}^{0}[G]$. 
Note that the decryption algorithm of the above construction is sequential. We can parallelize it (without harming the parallelization of the encryption) at the expense of strengthening the assumption we use.

Claim 4.6. Let PG (resp. LG) be a polynomial-stretch (resp. linear-stretch) $P R G$. Then for every polynomial $p(\cdot)$ there exists a $p(\cdot)$-one-time symmetric encryption scheme $(E, D)$ such that $E \in \mathrm{NC}^{0}[P G]$ and $D \in \mathrm{NC}^{0}[P G]$ (resp. $E \in \mathrm{NC}^{0}[L G]$ and $\left.D \in \mathrm{NC}^{1}[L G]\right)$.

Proof. Use Construction 4.3 (where $\left|r_{i}\right|=\left|G\left(r_{i-1}\right)\right|$ ). When the stretch of $G$ is polynomial (resp. linear) the construction requires only $O(1)$ (resp. $O(\log k))$ invocations of $G$, and therefore, so does the decryption algorithm.

4.2. From one-time encryption to computational encoding. Let $f=$ $\left\{f_{n}:\{0,1\}^{n} \rightarrow\{0,1\}^{\ell(n)}\right\}_{n \in \mathbb{N}}$ be a polynomial-time computable function, computed by the uniform circuit family $\left\{C_{n}\right\}_{n \in \mathbb{N}}$. We use a one-time symmetric encryption scheme $(E, D)$ as a black box to encode $f$ by a perfectly correct computational encoding $\hat{f}=\left\{\hat{f}_{n}\right\}_{n \in \mathbb{N}}$. Each $\hat{f}_{n}$ will be an $\mathrm{NC}^{0}$ circuit with an oracle access to the encryption algorithm $E$, where the latter is viewed as a function of the key, the message, and its random coin tosses. The construction uses a variant of Yao's garbled circuit technique (Yao 1986). Our notation and terminology for this section borrow from previous presentations of Yao's construction in Lindell \& Pinkas (2004); Naor et al. (1999); Rogaway (1991). Security proofs for variants of this construction were given implicitly in Rogaway (1991), Tate \& Xu (2003) and Lindell \& Pinkas (2004) in the context of secure computation. However, they cannot be directly used in our context for different reasons. (In particular, the analysis of Lindell \& Pinkas relies on a special form of symmetric encryption and does not achieve perfect correctness, while that of Rogaway and Tate \& Xu relies on a linear-stretch PRG.) Before we describe the actual encoding it will be convenient to think of the following "physical" analog that uses locks and boxes.

A physical encoding. To each wire of the circuit we assign a pair of keys: a 0 -key that represents the value 0 and a 1-key that represents the value 1 . For each of these pairs we randomly color one key black and the other key white. This way, given a key one cannot tell which bit it represents (since the coloring is random). For every gate of the circuit, the encoding consists of four double-locked boxes: a white-white box (which is locked by the white keys of the wires that enter the gate), a white-black box (locked by the white key of the left incoming wire and the black key of the right incoming wire), a black-white 
box (locked by the black key of the left incoming wire and the white key of the right incoming wire) and a black-black box (locked by the black keys of the incoming wires). Inside each box we put one of the keys of the gate's output wires. Specifically, if a box is locked by the keys that represent the values $\alpha, \beta$ then for every outgoing wire we put in the box the key that represents the bit $g(\alpha, \beta)$, where $g$ is the function that the gate computes. For example, if the gate is an OR gate then the box which is locked by the incoming keys that represent the bits $(0,1)$ contains all the 1-keys of the outgoing wires. So if one has a single key for each of the incoming wires, he can open only one box and get a single key for each of the outgoing wires. Moreover, as noted before, holding these keys does not reveal any information about the bits they represent.

Now, fix some input $x$ for $f_{n}$. For each wire, exactly one of the keys corresponds to the value of the wire (induced by $x$ ); we refer to this key as the active key and to the second key as the inactive key. We include in the encoding of $f_{n}(x)$ the active keys of the input wires. (This is the only place in which the encoding depends on the input $x$.) Using these keys and the locked boxes as described above, one can obtain the active keys of all the wires by opening the corresponding boxes in a bottom-to-top order. To make this information useful (i.e., to enable decoding of $f_{n}(x)$ ), we append to the encoding the semantics of the output wires; namely, for each output wire we expose whether the 1-key is white or black. Hence, the knowledge of the active key of an output wire reveals the value of the wire.

The actual encoding. The actual encoding is analogous to the above physical encoding. We let random strings play the role of physical keys. Instead of locking a value in a double-locked box, we encrypt it under the XOR of two keys. Before formally defining the construction, we need the following notation. Denote by $x=\left(x_{1}, \ldots, x_{n}\right)$ the input for $f_{n}$. Let $k=k(n)$ be a security parameter which may be set to $n^{\varepsilon}$ for an arbitrary positive constant $\varepsilon$ (see Remark 3.3). Let $\Gamma(n)$ denote the number of gates in $C_{n}$. For every $1 \leq i \leq\left|C_{n}\right|$, denote by $b_{i}(x)$ the value of the $i$-th wire induced by the input $x$; when $x$ is clear from the context we simply use $b_{i}$ to denote the wire's value.

Our encoding $\hat{f}_{n}(x,(r, W))$ consists of random inputs of two types: $\left|C_{n}\right|$ pairs of strings $W_{i}^{0}, W_{i}^{1} \in\{0,1\}^{2 k}$, and $\left|C_{n}\right|$ bits (referred to as masks) denoted $r_{1}, \ldots, r_{\left|C_{n}\right|}$. (In fact, each application of the encryption scheme will use some additional random bits. To simplify notation, we keep these random inputs implicit.) The strings $W_{i}^{0}, W_{i}^{1}$ will serve as the 0-key and the 1-key of the $i$-th wire, while the bit $r_{i}$ will determine which of these keys is the black key. We 
use $c_{i}$ to denote the value of wire $i$ masked by $r_{i}$; namely, $c_{i}=b_{i} \oplus r_{i}$. Thus, $c_{i}$ is the color of the active key of the $i$-th wire (with respect to the input $x$ ). As before, the encoding $\hat{f}_{n}(x,(r, W))$ will reveal each active key $W_{i}^{b_{i}}$ and its color $c_{i}$ but will hide the inactive keys $W_{i}^{1-b_{i}}$ and the masks $r_{i}$ of all the wires (except the masks of the output wires). Intuitively, since the active keys and inactive keys are distributed identically, the knowledge of an active key $W_{i}^{b_{i}}$ does not reveal the value $b_{i}$.

The encoding $\hat{f}_{n}$ consists of the concatenation of $O\left(\left|C_{n}\right|\right)$ functions, which include several entries for each gate and for each input and output wire. In what follows, $\oplus$ denotes bitwise-xor on strings; when we want to emphasize that the operation is applied to single bits we will usually denote it by either + or - . We use $\circ$ to denote concatenation. For every $\beta \in\{0,1\}$ and every $i$, we view the string $W_{i}^{\beta}$ as if it is partitioned into two equal-size parts denoted $W_{i}^{\beta, 0}, W_{i}^{\beta, 1}$.

Construction 4.7. Let $C_{n}$ be a circuit that computes $f_{n}$. Then we define $\hat{f}_{n}(x,(r, W))$ to be the concatenation of the following functions of $(x,(r, W))$.

Input wires: For an input wire $i$, labeled by a literal $\ell$ (either some variable $x_{u}$ or its negation), we append the function $W_{i}^{\ell} \circ\left(\ell+r_{i}\right)$.

Gates: Let $t \in[\Gamma(n)]$ be a gate that computes the function $g \in\{\mathrm{AND}, \mathrm{OR}\}$ with input wires $i, j$ and output wires $y_{1}, \ldots, y_{m}$. We associate with this gate four functions that are referred to as gate labels. Specifically, for each of the four choices of $a_{i}, a_{j} \in\{0,1\}$, we define a corresponding function $Q_{t}^{a_{i}, a_{j}}$. This function can be thought of as the box whose color is $\left(a_{i}, a_{j}\right)$. It is defined as follows:

$$
\begin{array}{r}
Q_{t}^{a_{i}, a_{j}}(r, W) \stackrel{\text { def }}{=} E_{W_{i}^{a_{i}-r_{i}, a_{j}} \oplus W_{j}^{a_{j}-r_{j}, a_{i}}}\left(W_{y_{1}}^{g\left(a_{i}-r_{i}, a_{j}-r_{j}\right)} \circ\left(g\left(a_{i}-r_{i}, a_{j}-r_{j}\right)+r_{y_{1}}\right) \circ \cdots\right. \\
\left.\circ W_{y_{m}}^{g\left(a_{i}-r_{i}, a_{j}-r_{j}\right)} \circ\left(g\left(a_{i}-r_{i}, a_{j}-r_{j}\right)+r_{y_{m}}\right)\right),
\end{array}
$$

where $E$ is a one-time symmetric encryption algorithm. (For simplicity, the randomness of $E$ is omitted.) That is, the colored keys of all the output wires of this gate are encrypted under a key that depends on the keys of the input wires of the gate. Note that $Q_{t}^{a_{i}, a_{j}}$ depends only on the random inputs. We refer to the label $Q_{t}^{c_{i}, c_{j}}$ that is indexed by the colors of the active keys of the input wires as an active label, and to the other three labels as the inactive labels.

Output wires: For each output wire $i$ of the circuit, we add the mask $r_{i}$ of this wire. 
It is not hard to verify that $\hat{f}_{n}$ is in $\mathrm{NC}^{0}[E]$. In particular, a term of the form $W_{i}^{\ell}$ is a 3 -local function of $W_{i}^{0}, W_{i}^{1}$ and $\ell$, since its $j$-th bit depends on the $j$-th bit of $W_{i}^{0}$, the $j$-th bit of $W_{i}^{1}$ and on the literal $\ell$. Similarly, the keys that are used in the encryptions are 8-local functions, and the arguments to the encryption are 6-local functions of $(r, W)$.

We will now analyze the complexity of $\hat{f}_{n}$. The output complexity and randomness complexity of $\hat{f}$ are both dominated by the complexity of the gate labels. Generally, the complexity of these functions is $\operatorname{poly}\left(\left|C_{n}\right| \cdot k\right)$ (since the encryption $E$ is computable in polynomial time). However, when the circuit $C_{n}$ has bounded fan-out (say 2 ) each invocation of the encryption uses poly $(k)$ random bits and outputs poly $(k)$ bits. Hence, the overall complexity is $O\left(\left|C_{n}\right|\right)$. poly $(k)=O\left(\left|C_{n}\right| \cdot n^{\varepsilon}\right)$ for an arbitrary constant $\varepsilon>0$. Since any circuit with unbounded fan-out of size $\left|C_{n}\right|$ can be (efficiently) transformed into a boundedfanout circuit whose size is $O\left(\left|C_{n}\right|\right.$ ) (at the price of a logarithmic factor in the depth), we get an encoding of size $O\left(\left|C_{n}\right| \cdot n^{\varepsilon}\right)$ for every (unbounded fan-out) circuit family $\left\{C_{n}\right\}$.

Let $\mu(n), s(n)$ be the randomness complexity and the output complexity of $\hat{f}_{n}$ respectively. We claim that the function family $\hat{f}=\left\{\hat{f}_{n}:\{0,1\}^{n} \times\right.$ $\left.\{0,1\}^{\mu(n)} \rightarrow\{0,1\}^{s(n)}\right\}_{n \in \mathbb{N}}$ defined above is indeed a computationally randomized encoding of the family $f$. We start with perfect correctness.

Lemma 4.8 (Perfect correctness). There exists a polynomial-time decoder algorithm $B$ such that for every $n \in \mathbb{N}$ and every $x \in\{0,1\}^{n}$ and $(r, W) \in$ $\{0,1\}^{\mu(n)}$, we have $B\left(1^{n}, \hat{f}_{n}(x,(r, W))\right)=f_{n}(x)$.

Proof. $\quad$ Let $\alpha=\hat{f}_{n}(x,(r, W))$ for some $x \in\{0,1\}^{n}$ and $(r, W) \in\{0,1\}^{\mu(n)}$. Given $\alpha$, our decoder computes, for every wire $i$, the active key $W_{i}^{b_{i}}$ and its color $c_{i}$. Then, for an output wire $i$, the decoder retrieves the mask $r_{i}$ from $\alpha$ and computes the corresponding output bit of $f_{n}(x)$; i.e., outputs $b_{i}=c_{i}-r_{i}$. (Recall that the masks of the output wires are given explicitly as part of $\alpha$.) The active keys and their colors are computed by scanning the circuit from bottom to top.

For an input wire $i$ the desired value, $W_{i}^{b_{i}} \circ c_{i}$, is given as part of $\alpha$. Next, consider a wire $y$ that goes out of a gate $t$, and assume that we have already computed the desired values of the input wires $i, j$ of this gate. We use the colors $c_{i}, c_{j}$ of the active keys of the input wires to select the active label $Q_{t}^{c_{i}, c_{j}}$ of the gate $t$ (and ignore the other three inactive labels of this gate). Consider this label as in Construction 4.7; recall that this cipher was encrypted under the key $W_{i}^{c_{i}-r_{i}, c_{j}} \oplus W_{j}^{c_{j}-r_{j}, c_{i}}=W_{i}^{b_{i}, c_{j}} \oplus W_{j}^{b_{j}, c_{i}}$. Since we have already computed 
the values $c_{i}, c_{j}, W_{i}^{b_{i}}$ and $W_{j}^{b_{j}}$, we can decrypt the label $Q_{t}^{c_{i}, c_{j}}$ (by applying the decryption algorithm $D$ ). Hence, we can recover the encrypted plaintext, that includes, in particular, the value $W_{y}^{g\left(b_{i}, b_{j}\right)} \circ\left(g\left(b_{i}, b_{j}\right)+r_{y}\right)$, where $g$ is the function that gate $t$ computes. Since by definition $b_{y}=g\left(b_{i}, b_{j}\right)$, the decrypted string contains the desired value.

REMARK 4.9. By the description of the decoder it follows that if the circuit $C_{n}$ is in $\mathrm{NC}^{i}$, then the decoder is in $\mathrm{NC}^{i}[D]$, where $D$ is the decryption algorithm. In particular if $D$ is in $\mathrm{NC}^{j}$ then the decoder is in $\mathrm{NC}^{i+j}$. This fact will be useful for some of the applications discussed in Section 5.

To deduce computational privacy we need to prove the following lemma, whose proof is deferred to Section 6 .

Lemma 4.10 (Computational privacy). There is a probabilistic polynomialtime simulator $S$ such that for any family of strings $\left\{x_{n}\right\}_{n \in \mathbb{N}}$ with $\left|x_{n}\right|=n$, we have $S\left(1^{n}, f_{n}\left(x_{n}\right)\right) \stackrel{c}{\equiv} \hat{f}_{n}\left(x_{n}, U_{\mu(n)}\right)$.

REMARK 4.11 (Information-theoretic variant). Construction 4.7 can be instantiated with a perfect (information-theoretic) encryption scheme, yielding a perfectly private randomized encoding. (The privacy proof given in Section 6 can be easily modified to treat this case.) However, in such an encryption the key must be as long as the encrypted message (Shannon 1949). It follows that the wires' key length grows exponentially with their distance from the outputs, rendering the construction efficient only for $\mathrm{NC}^{1}$ circuits. This informationtheoretic variant of the garbled circuit construction was previously suggested in Ishai \& Kushilevitz (2002). We will use it in Section 4.3 to obtain a computational encoding with a parallel decoder.

4.3. Main results. Combining Lemmas 4.8, 4.10, and 4.5 we get an $\mathrm{NC}^{0}$ encoding of any efficiently computable function using an oracle to a minimal PRG.

TheOREM 4.12. Suppose $f$ is computed by a uniform family $\left\{C_{n}\right\}$ of polynomial-size circuits. Let $G$ be a (minimal) PRG. Then $f$ admits a perfectly correct computational encoding $\hat{f}$ in $\mathrm{NC}^{0}[G]$. The complexity of $\hat{f}$ is $O\left(\left|C_{n}\right| \cdot n^{\varepsilon}\right)$ (for an arbitrary constant $\varepsilon>0$ ).

We turn to the question of eliminating the PRG oracles. We follow the natural approach of replacing each oracle with an $\mathrm{NC}^{0}$ implementation. (A more 
general but less direct approach will be described in Remark 4.16.) Using Theorem 6.2 of Applebaum et al. (2004), a minimal PRG in $\mathrm{NC}^{0}$ is implied by a $\mathrm{PRG}$ in $\mathcal{P} \mathcal{R} \mathcal{E} \mathcal{N}$, and in particular by a $\mathrm{PRG}$ in $\mathrm{NC}^{1}$ or even uniform $\oplus \mathrm{L} /$ poly. Thus, we can base our main theorem on the following "easy PRG" assumption.

Assumption 4.13 (Easy PRG (EPRG)). There exists a PRG in uniform $\oplus \mathrm{L} /$ poly.

As discussed in Section 1.1, EPRG is a very mild assumption. In particular, it is implied by most standard cryptographic intractability assumptions, and is also implied by the existence in uniform $\oplus \mathrm{L} /$ poly of one-way permutations and other types of one-way functions.

Combining Theorem 4.12 with the EPRG assumption, we get a computational encoding in $\mathrm{NC}^{0}$ for every efficiently computable function. To optimize its parameters we apply a final step of perfect encoding, yielding a computational encoding with degree 3 and locality 4 (see Remark 3.6). Thus, we get the following main theorem.

Theorem 4.14. Suppose $f$ is computed by a uniform family $\left\{C_{n}\right\}$ of polynomial-size circuits. Then, under the EPRG assumption, $f$ admits a perfectly correct computational encoding $\hat{f}$ of degree 3 , locality 4 and complexity $O\left(\left|C_{n}\right| \cdot n^{\varepsilon}\right)$ (for an arbitrary constant $\varepsilon>0$ ).

Corollary 4.15. Under the EPRG assumption, $\mathcal{C} \mathcal{R} \mathcal{E N}=\mathrm{BPP}$.

Proof. Let $f(x)$ be a function in BPP. It follows that there exists a function $f^{\prime}(x, z) \in \mathrm{P}$ such that $\operatorname{Pr}_{z}\left[f^{\prime}(x, z) \neq f(x)\right] \leq 2^{-n}$ for every $x \in\{0,1\}^{n}$. Let $\hat{f}^{\prime}((x, z), r)$ be the $\mathrm{NC}^{0}$ computational encoding of $f^{\prime}$ promised by Theorem 4.14. Since $f^{\prime}$ is a statistical encoding of $f$ (the simulator and the decoder are simply the identity functions), it follows from Lemma 3.5 that $\hat{f}(x,(z, r)) \stackrel{\text { def }}{=} \hat{f}^{\prime}((x, z), r)$ is a computational encoding of $f$ in $\mathrm{NC}^{0}$.

Conversely, suppose $f \in \mathcal{C} \mathcal{R E N}$ and let $\hat{f}$ be an $\mathrm{NC}^{0}$ computational encoding of $f$. A BPP algorithm for $f$ can be obtained by first computing $\hat{y}=\hat{f}(x, r)$ on a random $r$ and then invoking the decoder on $\hat{y}$ to obtain the output $y=f(x)$ with high probability.

REMARK 4.16 (Relaxing the EPRG assumption). The EPRG assumption is equivalent to the existence of a $\mathrm{PRG}$ in $\mathrm{NC}^{0}$ or in $\mathcal{P} \mathcal{R E N}$. It is possible to base Theorem 4.14 on a seemingly more liberal assumption by taking an 
alternative approach that does not rely on a perfect encoding. The idea is to first replace each PRG oracle with an implementation $G$ from some class $\mathcal{C}$, and only then apply a (perfectly correct) statistical encoding to the resulting $\mathrm{NC}^{0}[G]$ circuit. Thus, we need $G$ to be taken from a class $\mathcal{C}$ such that $\mathrm{NC}^{0}[\mathcal{C}] \subseteq \mathcal{S} \mathcal{R} \mathcal{E} \mathcal{N}$. It follows from Applebaum et al. (2004) and Ishai \& Kushilevitz (2002) that the class uniform NL/poly has this property (and furthermore, functions in uniform $\mathrm{NL} /$ poly admit a statistical $\mathrm{NC}^{0}$ encoding with perfect correctness). Thus, we can replace uniform $\oplus \mathrm{L} /$ poly in the EPRG assumption with uniform NL/poly. Another alternative is to assume the existence of a onetime symmetric encryption $(E, D)$ whose encrypting algorithm $E$ is in $\mathcal{S R E N}$. According to Applebaum et al. (2004) the last assumption is equivalent to the existence of one-time symmetric encryption (with negligible decryption error) whose encryption algorithm is in $\mathrm{NC}^{0}$. Hence by plugging this scheme to Construction 4.7, we obtain a computationally private, statistically correct randomized encoding in $\mathrm{NC}^{0}$ for any polynomial-time computable function (and in particular derive Corollary 4.15). In fact, we can even obtain perfect correctness (as in Theorem 4.14) by assuming the existence of one-time symmetric encryption in uniform NL/poly (which, using Applebaum et al. 2004 and Ishai \& Kushilevitz 2002, implies such an errorless scheme in $\mathrm{NC}^{0}$ ). Note that $(\mathrm{PRG} \in$ uniform $\oplus \mathrm{L} /$ poly $) \Rightarrow(\mathrm{PRG} \in$ uniform NL/poly) $\Rightarrow$ (one-time encryption $\in$ uniform NL/poly), while the converse implications are not known to hold.

On the parallel complexity of the decoder. As we shall see in Section 5, it is sometimes useful to obtain a computational encoding whose decoder is also parallelized. Recall that if the circuit computing $f$ is an $\mathrm{NC}^{i}$ circuit and the decryption algorithm (used in the construction) is in $\mathrm{NC}^{j}$, we obtain a parallel decoder in $\mathrm{NC}^{i+j}$ (see Remark 4.9). Unfortunately, we cannot use the parallel symmetric encryption scheme of Construction 4.3 for this purpose because of its sequential decryption.

We can get around this problem by strengthening the EPRG assumption. Suppose we have a polynomial-stretch PRG in $\mathrm{NC}^{1}$. (This is implied by some standard cryptographic assumptions, see Naor \& Reingold 2004.) In such a case, by Claim 4.6, we can obtain a one-time symmetric encryption scheme $(E, D)$ (for messages of a fixed polynomial length) in which both $E$ and $D$ are in $\mathrm{NC}^{1}$. Our goal is to turn this into a scheme $(\hat{E}, \hat{D})$ in which the encryption $\hat{E}$ is in $\mathrm{NC}^{0}$ and the decryption is still in $\mathrm{NC}^{1}$. We achieve this by applying to $(E, D)$ the encoding given by the information-theoretic variant of the garbled circuit construction (see Remark 4.11 or Ishai \& Kushilevitz 2002). That is, 
$\hat{E}$ is a (perfectly correct and private) $\mathrm{NC}^{0}$ encoding of $E$, and $\hat{D}$ is obtained by composing $D$ with the decoder of the information-theoretic garbled circuit. (The resulting scheme $(\hat{E}, \hat{D})$ is still a secure encryption scheme, see Applebaum et al. 2004 or Lemma 5.3.) Since the symmetric encryption $\left(E^{\prime}, D^{\prime}\right)$ employed by the information-theoretic garbled circuit is in $\mathrm{NC}^{0}$, its decoder can be implemented in $\mathrm{NC}^{1}\left[D^{\prime}\right]=\mathrm{NC}^{1}$. Thus, $\hat{D}$ is also in $\mathrm{NC}^{1}$ (as $\mathrm{NC}^{0}[$ decoder $]=\mathrm{NC}^{1}$ ). Combining this encryption scheme with Construction 4.7, we get a computational encoding of a function $f \in \mathrm{NC}^{i}$ with encoding in $\mathrm{NC}^{0}$ and decoding in $\mathrm{NC}^{i+1}$. Assuming there exists a linear-stretch PRG in $\mathrm{NC}^{1}$, we can use a similar argument to obtain an $\mathrm{NC}^{0}$ encoding for $f$ whose decoding in $\mathrm{NC}^{i+2}$. (In this case we use the linear-PRG part of Claim 4.6.) Summarizing, we have the following:

Claim 4.17. Suppose there exists a PRG with polynomial stretch (resp. linear stretch) in $\mathrm{NC}^{1}$. Then every function $f \in \mathrm{NC}^{i}$ admits a perfectly correct computational encoding in $\mathrm{NC}^{0}$ whose decoder is in $\mathrm{NC}^{i+1}$ (resp. $\mathrm{NC}^{i+2}$ ).

\section{Applications}

5.1. Relaxed assumptions for cryptography in $\mathrm{NC}^{0}$. In Applebaum et al. (2004) it was shown that, under relatively mild assumptions, many cryptographic tasks can be implemented in $\mathrm{NC}^{0}$. This was proved by arguing that: (1) the security of most primitives is inherited by their statistical or perfect randomized encoding; and (2) statistical or perfect encodings can be obtained for functions in relatively high complexity classes such as $\mathrm{NC}^{1}$, uniform $\oplus \mathrm{L} /$ poly or uniform NL/poly. Thus, if a primitive $\mathcal{P}$ can be computed in these classes, then it can also be computed in $\mathrm{NC}^{0}$.

In this work, we consider primitives whose security is also inherited by their computational encoding. (In some cases we will need to rely on perfect correctness, which we get "for free" in our main construction.) It follows from Theorem 4.14 that, under the EPRG assumption, any such primitive $\mathcal{P}$ can be computed in $\mathrm{NC}^{0}$ if it exists at all (i.e., can be computed in polynomial time).

Some primitives, such as collision resistant hash functions and one-way permutations, do not respect computational encoding. However, many others do. These include public-key encryption, (stateless) symmetric encryption, commitments, signatures, message authentication schemes (MACs), and zero knowledge proofs. (See Goldreich 2001, 2004 for detailed definitions of these cryptographic primitives.) In all these cases, we can replace the sender (i.e., the encrypting party, committing party, signer or prover, according to the case) with its computational encoding and let the receiver (the decrypting party or 
verifier) use the decoding algorithm to translate the output of the new sender to an output of the original one. The security of the resulting scheme reduces to the security of the original one by using the efficient simulator. These reductions are analogous to those given in Applebaum et al. (2004) for the case of statistical encoding. For completeness, we provide a full treatment for encryption schemes and sketch the proofs for commitments, signatures, and zero-knowledge proofs. We also show how to use the EPRG assumption to construct an instance-hiding scheme between an $\mathrm{NC}^{0}$ user and a BPP-oracle.

Definition 5.1. A secure public-key encryption scheme (PKE) is a triple $(G, E, D)$ of probabilistic polynomial-time algorithms satisfying the following conditions:

- Viability: On input $1^{n}$ the key generation algorithm, $G$, outputs a pair of keys $(e, d)$. For every pair $(e, d)$ such that $(e, d) \in G\left(1^{n}\right)$, and for every plaintext $x \in\{0,1\}^{*}$, the algorithms $E, D$ satisfy

$$
\operatorname{Pr}[D(d, E(e, x)) \neq x)] \leq \varepsilon(n)
$$

where $\varepsilon(n)$ is a negligible function and the probability is taken over the internal coin tosses of algorithms $E$ and $D$.

- Security: For every polynomial $\ell(\cdot)$, and any families of plaintexts $\left\{x_{n}\right\}_{n \in \mathbb{N}}$ and $\left\{x_{n}^{\prime}\right\}_{n \in \mathbb{N}}$ where $x_{n}, x_{n}^{\prime} \in\{0,1\}^{\ell(n)}$, we have

$$
\left(e \leftarrow G_{1}\left(1^{n}\right), E\left(e, x_{n}\right)\right) \stackrel{c}{\equiv}\left(e \leftarrow G_{1}\left(1^{n}\right), E\left(e, x_{n}^{\prime}\right)\right),
$$

where $G_{1}\left(1^{n}\right)$ denotes the first element in the pair $G\left(1^{n}\right)$.

The definition of a private-key encryption scheme is similar, except that the public key is omitted from the ensembles. That is, instead of (5.2) we require that $E\left(G_{1}\left(1^{n}\right), x_{n}\right) \stackrel{c}{\equiv} E\left(G_{1}\left(1^{n}\right), x_{n}^{\prime}\right)$. An extension to multiple-message security, where the indistinguishability requirement should hold for encryptions of polynomially many messages, follows naturally (see Goldreich 2004, Chapter 5 for formal definitions). In the public-key case, multiple-message security is implied by single-message security as defined above, whereas in the private-key case it is a strictly stronger notion. In the following we explicitly address only the (single-message) public-key case, but the treatment easily holds for the case of private-key encryption with multiple-message security. 
Lemma 5.3. Let $\mathcal{E}=(G, E, D)$ be a secure public-key encryption scheme, where $E(e, x, r)$ is viewed as a polynomial-time computable function that encrypts the message $x$ using the key $e$ and randomness $r$. Let $\hat{E}((e, x),(r, s))=$ $\hat{E}((e, x, r), s)$ be a computational randomized encoding of $E$ and let $\hat{D}(d, \hat{y}) \stackrel{\text { def }}{=}$ $D(d, B(\hat{y}))$ be the composition of $D$ with the decoder $B$ of $\hat{E}$. Then the scheme $\hat{\mathcal{E}} \stackrel{\text { def }}{=}(G, \hat{E}, \hat{D})$ is also a secure public-key encryption scheme.

Proof. The uniformity of the encoding guarantees that the functions $\hat{E}$ and $\hat{D}$ can be efficiently computed. The viability of $\hat{\mathcal{E}}$ follows in a straightforward way from the correctness of the decoder $B$. Indeed, if $(e, d)$ are in the support of $G\left(1^{n}\right)$, then for any plaintext $x$ we have

$$
\begin{aligned}
\operatorname{Pr}_{r, s}[\hat{D}(d, \hat{E}(e, x, r, s)) \neq x]= & \operatorname{Pr}_{r, s}[D(d, B(\hat{E}(e, x, r, s))) \neq x] \\
\leq & \operatorname{Pr}_{r, s}[B(\hat{E}((e, x, r), s)) \neq E(e, x, r)] \\
& +\operatorname{Pr}_{r}[D(d, E(e, x, r)) \neq x] \\
\leq & \varepsilon(n),
\end{aligned}
$$

where $\varepsilon(\cdot)$ is negligible in $n$ and the probabilities are also taken over the coin tosses of $D$; the first inequality follows from the union bound and the second from the viability of $\mathcal{E}$ and the statistical correctness of $\hat{E}$.

We move on to prove the security of the construction. Let $S$ be the efficient computational simulator of $\hat{E}$. Then, for every polynomial $\ell(\cdot)$, and every families of plaintexts $\left\{x_{n}\right\}_{n \in \mathbb{N}}$ and $\left\{x_{n}^{\prime}\right\}_{n \in \mathbb{N}}$ where $x_{n}, x_{n}^{\prime} \in\{0,1\}^{\ell(n)}$, we have

$$
\begin{aligned}
\left(e \leftarrow G_{1}\left(1^{n}\right), \hat{E}\left(e, x_{n}, r_{n}, s_{n}\right)\right) & \stackrel{c}{\equiv}\left(e \leftarrow G_{1}\left(1^{n}\right), S\left(E\left(e, x_{n}, r_{n}\right)\right)\right) \\
& \stackrel{\equiv}{\equiv}\left(e \leftarrow G_{1}\left(1^{n}\right), S\left(E\left(e, x_{n}^{\prime}, r_{n}\right)\right)\right) \\
& \stackrel{c}{\equiv}\left(e \leftarrow G_{1}\left(1^{n}\right), \hat{E}\left(e, x_{n}^{\prime}, r_{n}, s_{n}\right)\right),
\end{aligned}
$$

where $r_{n}$ and $s_{n}$ are uniformly chosen random strings of an appropriate length. The first and third transitions follow from the privacy of $\hat{E}$ and Fact 2.5, and the second is due to the security of $\mathcal{E}$ and Fact 2.3. Hence, the security of $\hat{\mathcal{E}}$ follows from the transitivity of the relation $\stackrel{c}{\equiv}$ (Fact 2.1).

In particular, if the scheme $\mathcal{E}=(G, E, D)$ enables errorless decryption and the encoding $\hat{E}$ is perfectly correct, then the scheme $\hat{\mathcal{E}}$ also enables errorless decryption. Additionally, the above lemma is easily extended to the case of private-key encryption with multiple-message security. 
Signatures. Let $\mathcal{S}=(G, S, V)$ be a signature scheme, where $G$ is a keygeneration algorithm that generates the signing and verification keys $(s, v)$, the signing function $S(s, \alpha, r)$ computes a signature $\beta$ on the document $\alpha$ using the key $s$ and randomness $r$, and the verification algorithm $V(v, \alpha, \beta)$ verifies that $\beta$ is a valid signature on $\alpha$ using the verification key $v$. The three algorithms run in probabilistic polynomial time, and the scheme provides correct verification for legal signatures (ones that were produced by the signing function using the corresponding signing key). The scheme is secure (unforgeable) if it is infeasible to forge a signature in a chosen message attack. Specifically, any polynomial-time adversary that gets the verification key and an oracle access to the signing process $S(s, \cdot)$ fails (except with negligible probability) to produce a valid signature $\beta$ on a document $\alpha$ (with respect to the corresponding verification key $v$ ) for which it has not requested a signature from the oracle. (When the signing algorithm is probabilistic, the attacker does not have an access to the random coin tosses of the signing algorithm.)

Let $\hat{S}$ be a computational randomized encoding of $S$, and let $\hat{V}(v, \alpha, \hat{\beta}) \stackrel{\text { def }}{=}$ $V(v, \alpha, B(\hat{\beta}))$ be the composition of $V$ with the decoder $B$ of the encoding $\hat{S}$. We claim that the scheme $\hat{\mathcal{S}} \stackrel{\text { def }}{=}(G, \hat{S}, \hat{V})$ is also a signature scheme. The efficiency and correctness of $\hat{\mathcal{S}}$ follow from the uniformity of the encoding and its correctness. To prove the security of the new scheme we use the simulator to transform an attack on $\hat{\mathcal{S}}$ into an attack on $\mathcal{S}$. Specifically, given an adversary $\hat{A}$ that breaks $\hat{\mathcal{S}}$, we can break $\mathcal{S}$ by invoking $\hat{A}$ and emulating the oracle $\hat{S}$ using the simulator of the encoding and the signature oracle $S$. By Fact 2.4 the output of $\hat{A}$ when interacting with the emulated oracle is computationally indistinguishable from $\hat{A}$ 's output when interacting with the actual signing oracle $\hat{S}(s, \cdot)$. Moreover, if the forged signature $(\alpha, \hat{\beta})$ produced by $\hat{A}$ is valid under $\hat{\mathcal{S}}$, then it is translated into a valid signature $(\alpha, \beta)$ under $\mathcal{S}$ by using the decoder, i.e., $\beta=B(\hat{\beta})$. Hence, if the scheme $\hat{\mathcal{S}}$ can be broken with nonnegligible probability, then so can the scheme $\mathcal{S}$. A similar argument holds also in the private-key setting (i.e., in the case of MACs).

Commitments. A commitment scheme enables one party (a sender) to commit itself to a value while keeping it secret from another party (the receiver). Later, the sender can reveal the committed value to the receiver, and it is guaranteed that the revealed value is equal to the one determined at the commit stage. We start with the simple case of a non-interactive commitment. Such a scheme can be defined by a polynomial-time computable function $C(b, r)$ that outputs a commitment $c$ to the bit $b$ using the randomness $r$. We assume, without loss of generality, that the scheme has a canonical decommit stage in 
which the sender reveals $b$ by sending $b$ and $r$ to the receiver, who verifies that $C(b, r)$ is equal to the commitment $c$. The scheme should be both (computationally) hiding and (perfectly) binding. Hiding requires that $c=C(b, r)$ keeps $b$ computationally secret, that is, $C\left(0, U_{n}\right) \stackrel{c}{\equiv} C\left(1, U_{n}\right)$. Binding means that it is impossible for the sender to open its commitment in two different ways; that is, there are no $r_{0}$ and $r_{1}$ such that $C\left(0, r_{0}\right)=C\left(1, r_{1}\right)$.

Let $\hat{C}(b, r, s)$ be a perfectly correct, computationally private encoding of $C(b, r)$. Then $\hat{C}$ defines a computationally hiding, perfectly binding, non-interactive commitment. Hiding follows from the privacy of the encoding, as argued for the case of encryption in Lemma 5.3. Namely,

$$
\hat{C}(0, r, s) \stackrel{c}{\equiv} S(C(0, r, s)) \stackrel{c}{\equiv} S(C(1, r, s)) \stackrel{c}{\equiv} \hat{C}(1, r, s)
$$

where $r$ and $s$ are uniformly chosen strings of an appropriate length (the first and third transitions follow from the privacy of $\hat{C}$ and Fact 2.5, while the second transition follows from the hiding of $C$ and Fact 2.3). The binding property of $\hat{C}$ follows from the perfect correctness; namely, if there exists an ambiguous pair $\left(r_{0}, r_{0}^{\prime}\right),\left(r_{1}, r_{1}^{\prime}\right)$ such that $\hat{C}\left(0, r_{0}, s_{0}\right)=\hat{C}\left(1, r_{1}, s_{1}\right)$, then by perfect correctness $C\left(0, r_{0}\right)=C\left(1, r_{1}\right)$, which contradicts the binding of the original scheme. So when the encoding is in $\mathrm{NC}^{0}$ we get a commitment scheme whose sender is in $\mathrm{NC}^{0}$. It should be noted that the resulting scheme is computationally hiding even if the original scheme is unconditionally hiding. This is contrasted with the case of statistically private encodings as discussed in Applebaum et al. (2004).

In fact, in contrast to the primitives described so far, here we also improve the parallel complexity at the receiver's end. Indeed, on input $\hat{c}, b, r, s$ the receiver's computation consists of computing $\hat{C}(b, r, s)$ and comparing the result to $\hat{c}$. Assuming $\hat{C}$ is in $\mathrm{NC}^{0}$, the receiver can be implemented by an $\mathrm{NC}^{0}$ circuit augmented with a single (unbounded fan-in) AND gate. We refer to this special type of $\mathrm{AC}^{0}$ circuit as an $\mathrm{AND}_{n} \circ \mathrm{NC}^{0}$ circuit. This extension of $\mathrm{NC}^{0}$ is necessary as the locality of the function $f$ that the receiver computes cannot be constant. To see this, consider a receiver who decides whether to accept the opening of the commitment according to the committed bit $b$ and a string $r$ of constant length. Such a receiver can efficiently find $b$ before the decommit stage by going over all possible $r$ 's and checking whether there exists an $r$ such that $f(b, r)=$ accept. Hence, the locality of the receiver's computation $f$ should be super-logarithmic.

While the existence of non-interactive commitment schemes is implied by the existence of a 1-1 OWF (Blum 1983, Goldreich 2001, Const. 4.4.2), it is not known how to construct such a scheme based on arbitrary OWF (or 
equivalently PRG). However, the existence of a OWF allows one to construct an interactive commitment scheme (Naor 1991). In particular, the PRG based commitment scheme of Naor (1991) has the following form: First the receiver chooses a random string $r \in\{0,1\}^{3 n}$ and sends it to the sender, then the sender that wishes to commit to the bit $b$ chooses a random string $s \in\{0,1\}^{n}$ and sends the value of the function $C(b, r, s)$ to the receiver. (The exact definition of the function $C(b, r, s)$ is not important in our context.) To decommit the sender sends the randomness $s$ and the bit $b$ and the receiver accepts if $C(b, r, s)$ equals the message he had received in the commit phase. Computational hiding requires that $(r, C(0, r, s)) \stackrel{c}{\equiv}(r, C(1, r, s))$ for any choice of $r$. Perfect binding requires that, except with negligible probability (over the randomness of the receiver $r$ ), there are no $s_{0}$ and $s_{1}$ such that $C\left(0, r, s_{0}\right)=C\left(1, r, s_{1}\right)$.

Again, if we replace $C$ by a computationally private, perfectly correct encoding $\hat{C}$, we get a (constant-round) interactive commitment scheme (this follows by combining the previous arguments with Fact 2.5). Moreover, as in the noninteractive case, when the encoding is in $\mathrm{NC}^{0}$ the receiver's computation in the decommit phase is in $\mathrm{AND}_{n} \circ \mathrm{NC}^{0}$, and so, since the receiver's computation in the commit phase is in $\mathrm{NC}^{0}$, we get an $\mathrm{AND}_{n} \circ \mathrm{NC}^{0}$ receiver. As an immediate application, we obtain a constant-round protocol for coin flipping over the phone (Blum 1983) between an $\mathrm{NC}^{0}$ circuit and an $\mathrm{AND}_{n} \circ \mathrm{NC}^{0}$ circuit under the EPRG assumption.

Zero-knowledge proofs. We move on to the case of non-interactive zero knowledge proofs (NIZK). Such proof systems are similar to standard zeroknowledge protocols except that interaction is traded for the use of a public random string $\sigma$ to which both the prover and the verifier have a read-only access. More formally, a NIZK (with an efficient prover) for an NP relation $R(x, w)$ is a pair of probabilistic polynomial-time algorithms $(P, V)$ with the following properties:

○ (Completeness) for every $(x, w) \in R, \operatorname{Pr}[V(x, \sigma, P(x, w, \sigma))=1]>2 / 3$;

○ (Soundness) for every $x \notin L_{R}$ (i.e., $x$ such that $\forall w,(x, w) \notin R$ ) and every prover algorithm $P^{*}$ we have $\operatorname{Pr}\left[V\left(x, \sigma, P^{*}(x, \sigma)\right)=1\right]<1 / 3$;

○ (Zero-knowledge) there exists a probabilistic polynomial-time simulator $M$ such that for every string sequence $\left\{\left(x_{n}, w_{n}\right)\right\}$ where $\left(x_{n}, w_{n}\right) \in R$ we have $\left\{\left(x_{n}, \sigma, P\left(x_{n}, w_{n}, \sigma\right)\right)\right\} \stackrel{c}{\equiv}\left\{M\left(x_{n}\right)\right\}$.

(In all the above, $\sigma$ is uniformly distributed over $\{0,1\}^{\text {poly }(|x|)}$.) 
Similarly to the previous cases, we can compile the prover into its computational randomized encoding $\hat{P}$, while the new verifier $\hat{V}$ uses the decoder $B$ to translate the prover's encoded message $\hat{y}$ to the corresponding message of the original prover, and then invokes the original verifier (i.e., $\hat{V}=V(x, \sigma, B(\hat{y}))$ ). The completeness and soundness of the new protocol follow from the correctness of the encoding. The zero-knowledge property follows from the privacy of the encoding. That is, to simulate the new prover we define a simulator $\hat{M}$ that invokes the simulator $M$ of the original scheme and then applies the simulator $S$ of the encoding to the third entry of $M$ 's output. By Fact 2.5 and the privacy of $\hat{P}$ we have $(x, \sigma, \hat{P}(x, w, \sigma, r)) \stackrel{\stackrel{c}{\equiv}}{\equiv}(x, \sigma, S(P(x, w, \sigma)))$ (where $r$ is the randomness of the encoding $\hat{P})$ while Fact 2.3 ensures that $(x, \sigma, S(P(x, w, \sigma))) \stackrel{c}{\equiv} \hat{M}(x)$.

The above construction generalizes to interactive ZK-proofs with an efficient prover. In this case, we can encode the prover's computation (viewed as a function of his input, the NP-witness he holds, his private randomness and all the messages he has received so far), while the new receiver uses the decoder to translate the messages and then invokes the original protocol. The resulting protocol is still a computational ZK proof. (The proof is similar to the case of NIZK above, but relies on Fact 2.4 instead of Fact 2.3.) The same construction works for ZK arguments (in which the soundness holds only against a computationally bounded cheating prover). However, the above transformation results in a computational ZK even if the original protocol was a statistical ZK proof system. Again, this contrasts with the transformations obtained by using statistically private randomized encoding described in Applebaum et al. (2004).

Instance hiding. An instance hiding scheme (IHS) allows a powerful machine (an oracle) to help a more limited user compute some function $f$ on the user's input $x$; the user wishes to keep his input private and so he cannot just send it to the machine. We assume that the user is an algorithm from a low complexity class WEAK whereas the oracle is from a higher complexity class STRONG. In a (non-adaptive, single-oracle) IHS the user first transforms his input $x$ into a (randomized) encrypted instance $y=E(x, r)$ and then asks the oracle to compute $z=g(y)$. The user should be able to recover the value of $f(x)$ from $z$ by applying a decryption algorithm $D(x, r, z)$ (where $D \in$ WEAK) such that $D(x, r, g(E(x, r)))=f(x)$. The hiding of the scheme requires that $E(x, r)$ keeps $x$ secret, i.e., for any string families $\left\{x_{n}\right\}$ and $\left\{x_{n}^{\prime}\right\}$ (where $\left|x_{n}\right|=\left|x_{n}^{\prime}\right|$ ), the ensembles $E\left(x_{n}, r\right)$ and $E\left(x_{n}^{\prime}, r\right)$ are indistinguishable with respect to functions in STRONG. The default setting of instance hiding considered in the literature refers to a probabilistic polynomial-time user and a computationally unbounded machine. (See Feigenbaum 1993 for a survey on IHS schemes.) 
The notion of randomized encoding naturally gives rise to IHS in the following way: Given $f$ we define a related function $h(x, r)=f(x) \oplus r$ (where $|r|=|f(x)|)$. Let $\hat{h}((x, r), s)$ be a randomized encoding of $h$ whose decoder is $B$. Then we define $E(x,(r, s))=\hat{h}((x, r), s), g(y)=B(y)$ and $D(x, r, z)=r \oplus z$. The correctness of the scheme follows from the correctness of the encoding. To prove the privacy note that, by Fact 2.5,

$$
\hat{h}\left(x_{n}, r, s\right) \stackrel{c}{\equiv} S\left(f\left(x_{n}\right) \oplus r\right) \equiv S\left(f\left(x_{n}^{\prime}\right) \oplus r\right) \stackrel{c}{\equiv} \hat{h}\left(x_{n}^{\prime}, r, s\right) .
$$

Hence, under the EPRG assumption, we can construct such a scheme where WEAK $=\mathrm{NC}^{0}$ and STRONG $=\mathrm{BPP}$ (or P if perfect correctness is required).

We summarize some consequences of the EPRG assumption obtained so far.

TheOREM 5.4. Suppose that the EPRG assumption holds. Then:

(i) If there exists a public-key encryption scheme (resp., NIZK with an efficient prover or constant-round ZK proof with an efficient prover for every $\mathrm{NP}$ relation), then there exists such a scheme in which the encryption (prover) algorithm is in $\mathrm{NC}_{4}^{0}$.

(ii) If there exists a non-interactive commitment scheme, then there exists such a scheme in which the sender is in $\mathrm{NC}_{4}^{0}$ and the receiver is in $\mathrm{AND}_{n}$ 。 $\mathrm{NC}^{0}$.

(iii) There exists a stateless symmetric encryption scheme (resp., digital signature, $M A C$, a constant-round $Z K$ argument for every language in NP) in which the encryption (signing, prover) algorithm is in $\mathrm{NC}_{4}^{0}$.

(iv) There exists a constant-round commitment scheme in which the sender is in $\mathrm{NC}_{4}^{0}$ and the receiver is in $\mathrm{AND}_{n} \circ \mathrm{NC}^{0}$.

(v) For every polynomial-time computable function we have a (non-adaptive single-oracle) IHS in which the user is in $\mathrm{NC}_{5}^{0}$ and the oracle is in $\mathrm{BPP}$.

Note that the existence of (stateless) symmetric encryption, signature, MAC, constant-round commitment scheme and constant-round ZK arguments for NP does not require any additional assumption other than EPRG. This is a consequence of the fact that they can all be constructed (in polynomial time) 
from a PRG (see Goldreich 2001, 2004). For these primitives, we obtain more general (unconditional) results in the next subsection.

Theorem 5.4 reveals an interesting phenomenon. It appears that several cryptographic primitives (e.g., symmetric encryption schemes, digital signatures and MACs) can be implemented in $\mathrm{NC}^{0}$ despite the fact that their standard constructions rely on pseudorandom functions (PRFs) (Goldreich et al. 1986), which cannot be computed even in $\mathrm{AC}^{0}$ (Linial et al. 1993). For such primitives, we actually construct a sequential PRF from the PRG (as in Goldreich et al. 1986), use it as a building block to obtain a sequential construction of the desired primitive (e.g., symmetric encryption), and finally reduce the parallel-time complexity of the resulting function using our machinery. Of course, the security of the PRF primitive itself is not inherited by its computational (or even perfect) encoding.

Parallelizing the receiver. As mentioned above, the computational encoding promised by Theorem 4.14 does not support parallel decoding. Thus, we get primitives in which the sender is in $\mathrm{NC}^{0}$ but the receiver is not known to be in $\mathrm{NC}$, even if we started with a primitive that has an $\mathrm{NC}$ receiver. The following theorem tries to partially remedy this state of affairs. Assuming the existence of a PRG with a good stretch in $\mathrm{NC}^{1}$, we can rely on Claim 4.17 to convert sender-receiver schemes in which both the receiver and the sender are in $\mathrm{NC}$ to ones in which the sender is in $\mathrm{NC}^{0}$ and the receiver is still in $\mathrm{NC}$. (Similarly, assuming a linear-stretch PRG in $\mathrm{NC}^{1}$, we can obtain, for every $\mathrm{NC}$ function, a non-adaptive single-oracle IHS in which the user is in $\mathrm{NC}^{0}$ and the oracle is in NC.)

Theorem 5.5. Let $\mathcal{X}=(G, S, R)$ be a sender-receiver cryptographic scheme whose security is respected by computational encoding (e.g., encryption, signature, MAC, commitment scheme, NIZK), where $G$ is a key-generation algorithm (in case the scheme has one), $S \in \mathrm{NC}^{s}$ is the algorithm of the sender and $R \in \mathrm{NC}^{r}$ is the algorithm of the receiver. Then:

- If there exists a polynomial-stretch PRG in $\mathrm{NC}^{1}$, then there exists a similar scheme $\hat{\mathcal{X}}=(G, \hat{S}, \hat{R})$ in which $\hat{S} \in \mathrm{NC}^{0}$ and $\hat{R} \in \mathrm{NC}^{\max \{s+1, r\}}$.

- If there exists a linear-stretch $P R G$ in $\mathrm{NC}^{1}$, then there exists a similar scheme $\hat{\mathcal{X}}=(G, \hat{S}, \hat{R})$ in which $\hat{S} \in \mathrm{NC}^{0}$ and $\hat{R} \in \mathrm{NC}^{\max \{s+2, r\}}$.

PROOF. If there exists a polynomial-stretch (resp. linear-stretch) PRG in $\mathrm{NC}^{1}$, then we can use Claim 4.17 and get a computational encoding $\hat{S}$ for $S$ in 
$\mathrm{NC}^{0}$ whose decoder $B$ is in $\mathrm{NC}^{s+1}$ (resp. $\mathrm{NC}^{s+2}$ ). As usual, the new receiver $\hat{R}$ uses $B$ to decode the encoding, and then applies the original receiver $R$ to the result. Thus, $\hat{R}$ is in $\mathrm{NC}^{\max \{s+1, r\}}$ (resp. $\mathrm{NC}^{\max \{s+2, r\}}$ ).

5.2. Parallel reductions between cryptographic primitives. In the previous section we showed that many cryptographic tasks can be performed in $\mathrm{NC}^{0}$ if they can be performed at all, relying on the assumption that an easy PRG exists. Although EPRG is a very reasonable assumption, it is natural to ask what types of parallel reductions between primitives can be guaranteed unconditionally. In particular, such reductions would have consequences even if there exists a PRG in, say, $\mathrm{NC}^{4}$.

In this section, we consider the types of unconditional reductions that can be obtained using the machinery of Section 4 . We focus on primitives that can be reduced to a PRG (equivalently, using Håstad et al. 1999, to a one-way function). We argue that for any such primitive $\mathcal{F}$, its polynomial-time reduction to a PRG can be collapsed into an $\mathrm{NC}^{0}$ reduction to a PRG. More specifically, we present an efficient "compiler" that takes the code of an arbitrary PRG $G$ and outputs a description of an $\mathrm{NC}^{0}$ circuit $C$, having oracle access to a function $G^{\prime}$, such that for any (minimal) PRG $G^{\prime}$ the circuit $C\left[G^{\prime}\right]$ implements $\mathcal{F}$.

A compiler as above proceeds as follows. Given the code of $G$, it first constructs a code for an efficient implementation $f$ of $\mathcal{F}$. (In case we are given an efficient black-box reduction from $\mathcal{F}$ to a PRG, this code is obtained by plugging the code of $G$ into this reduction.) Then, applying a constructive form of Theorem 4.12 to the code of $f$, the compiler obtains a code $\hat{f}$ of an $\mathrm{NC}^{0}$ circuit which implements $\mathcal{F}$ by making an oracle access to a PRG. This code of $\hat{f}$ defines the required $\mathrm{NC}^{0}$ reduction from $\mathcal{F}$ to a PRG, whose specification depends on the code of the given PRG $G$. Thus, the reduction makes a nonblack-box use of the PRG primitive, even if the polynomial-time reduction it is based on is fully black-box.

Based on the above we can obtain the following informal "meta-theorem":

Meta-Theorem 5.6. Let $\mathcal{F}$ be a cryptographic primitive whose security is respected by computational encoding. Suppose that $\mathcal{F}$ is polynomial-time reducible to a $P R G$. Then $\mathcal{F}$ is $\mathrm{NC}^{0}$ reducible to a (minimal) $P R G$.

Since a minimal PRG can be reduced in $\mathrm{NC}^{0}$ to one-way permutations or more general types of one-way functions (see Haitner et al. 2005; Håstad et al. 1999 and Remark 6.6 in the full version of Applebaum et al. 2004), the minimal PRG in the conclusion of the above theorem can be replaced by these primitives. 
Instantiating $\mathcal{F}$ by concrete primitives, we get the following corollary:

Corollary 5.7. Let $G$ be a PRG. Then:

- There exists a stateless symmetric encryption scheme (resp., digital signature or $\mathrm{MAC}$ ) in which the encryption (signing) algorithm is in $\mathrm{NC}^{0}[G]$.

- There exists a constant-round commitment scheme (resp., constant-round coin-flipping protocol) in which the sender (first party) is in $\mathrm{NC}^{0}[G]$ and the receiver (second party) is in $\mathrm{AND}_{n} \circ \mathrm{NC}^{0}[G]$.

- For every NP language, there exists a constant-round ZK argument in which the prover is in $\mathrm{NC}^{0}[G]$.

Note that items (iii), (iv) of Theorem 5.4 can be derived from the above corollary, up to the exact locality.

The above results can be used to improve the parallel complexity of some known reductions. For example, Naor (1991) shows a commitment scheme in which the sender is in $\mathrm{NC}^{0}[L G]$, where $L G$ is a linear-stretch PRG. By using his construction, we derive a commitment scheme in which the sender (resp. the receiver) is in $\mathrm{NC}^{0}[G]$ (resp. $\mathrm{AND}_{n} \circ \mathrm{NC}^{0}[G]$ ) where $G$ is a minimal PRG. Since it is not known how to reduce a linear-stretch PRG to a minimal PRG even in NC, we get a non-trivial parallel reduction.

Other interesting examples arise in the case of primitives that are based on PRFs, such as MACs, symmetric encryption, and identification (see Goldreich 2004; Goldreich et al. 1986; Naor \& Reingold 1999 for these and other applications of PRFs). Since the known construction of a PRF from a PRG is sequential (Goldreich et al. 1986), it was not known how to reduce these primitives in parallel to (even a polynomial-stretch) PRG. This fact motivated the study of parallel constructions of PRFs in Naor \& Reingold $(1999,2004)$. In particular, Naor \& Reingold (1999) introduce a new cryptographic primitive called a synthesizer (SYNTH), and show that PRFs can be implemented in $\mathrm{NC}^{1}[\mathrm{SYNTH}]$. This gives an $\mathrm{NC}^{1}$ reduction from cryptographic primitives such as symmetric encryption to synthesizers. By Corollary 5.7, these primitives are in fact $\mathrm{NC}^{0}$ reducible to a PRG. Since (even a polynomial-stretch) PRG can be implemented in $\mathrm{NC}^{0}[\mathrm{SYNTH}]$ while synthesizers are not known to be even in $\mathrm{NC}[\mathrm{PRG}]$, our results improve both the complexity of the reduction and the underlying assumption. It should be noted, however, that our reduction only improves the parallel-time complexity of the encrypting party, while the constructions of Naor \& Reingold (1999) yield $\mathrm{NC}^{1}$ reductions on both ends. 
In contrast to the above, we show that a synthesizer in $\mathrm{NC}^{i}$ can be used to implement encryption in $\mathrm{NC}^{i}$ with decryption in NC. First, we use Naor \& Reingold (1999) to construct an encryption scheme $(E, D)$ and a polynomialstretch PRG $G$ such that $E$ and $D$ are in $\mathrm{NC}^{1}[\mathrm{SYNTH}]=\mathrm{NC}^{i+1}$ and $G$ is in $\mathrm{NC}^{0}[\mathrm{SYNTH}]=\mathrm{NC}^{i}$. Next, by Claim 4.6 and Remark 4.9, we obtain an $\mathrm{NC}^{0}[G]=\mathrm{NC}^{i}$ computational encoding $\hat{E}$ for $E$ whose decoder $B$ is in $\mathrm{NC}^{2 i}$. (We first use Claim 4.6 to construct one-time symmetric encryption $(O E, O D)$ such that $O E$ and $O D$ are in $\mathrm{NC}^{0}[G]=\mathrm{NC}^{i}$. Then we encode $E$ by plugging $O E$ into Construction 4.7 and obtain an $\mathrm{NC}^{0}[O E]=\mathrm{NC}^{i}$ computational encoding $\hat{E}$ for $E$. By Remark 4.9 the decoder $B$ of $\hat{E}$ is in $\mathrm{NC}^{i}[O D]=\mathrm{NC}^{2 i}$.) To decrypt ciphers of $\hat{E}$ we invoke the decoder $B$, and then apply the original decryption algorithm $D$ to the result. Therefore, the decryption algorithm of our new scheme $\hat{D}$ is in $\mathrm{NC}^{\max (2 i, i+1)}$. The same transformation can be applied to any primitive which is $\mathrm{NC}^{0}$ reducible to $\mathrm{PRF}$ (such as identification schemes and MACs).

5.3. Secure multi-party computation. Secure multi-party computation (MPC) allows several parties to evaluate a function of their inputs in a distributed way, so that both the privacy of their inputs and the correctness of the outputs are maintained. These properties should hold, to the extent possible, even in the presence of an adversary who may corrupt at most $t$ parties. This is typically formalized by comparing the adversary's interaction with the real process, in which the uncorrupted parties run the specified protocol on their inputs, with an ideal function evaluation process in which a trusted party is employed. The protocol is said to be secure if whatever the adversary "achieves" in the real process it could have also achieved by corrupting the ideal process. A bit more precisely, it is required that for every adversary $A$ interacting with the real process there is an adversary $A^{\prime}$ interacting with the ideal process, such that outputs of these two interactions are indistinguishable from the point of view of an external environment. See, e.g., Canetti (2000, 2001) and Goldreich (2004) for more detailed and concrete definitions.

There are a variety of different models for secure computation. These models differ in the power of the adversary, the network structure, and the type of "environment" that tries to distinguish between the real process and the ideal process. In the information-theoretic setting, both the adversary and the distinguishing environment may be computationally unbounded, whereas in the computational setting they are both bounded to probabilistic polynomial time.

The notion of randomizing polynomials was originally motivated by the goal of minimizing the round complexity of MPC. The motivating observation 
of Ishai \& Kushilevitz (2000) was that the round complexity of most general protocols from the literature (e.g., those of Ben-Or et al. 1988; Chaum et al. 1988; Goldreich et al. 1987) is related to the algebraic degree of the function being computed. Thus, by reducing the task of securely computing $f$ to that of securely computing some related low-degree function, one can obtain roundefficient protocols for $f$.

Randomizing polynomials (or low-degree randomized encodings) provide precisely this type of reduction. More specifically, suppose that the input $x$ to $f$ is distributed between the parties, who all wish to learn the output $f(x)$. If $f$ is represented by a vector $\hat{f}(x, r)$ of degree- $d$ randomizing polynomials, then the secure computation of $f$ can be non-interactively reduced to that of $\hat{f}$, where the latter is viewed as a randomized function of $x$. This reduction only requires each party to invoke the decoder of $\hat{f}$ on its local output, obtaining the corresponding output of $f$. The secure computation of $\hat{f}$, in turn, can be non-interactively reduced to that of a related deterministic function $\hat{f}^{\prime}$ of the same degree $d$. The idea is to let $\hat{f}^{\prime}\left(x, r^{1}, \ldots, r^{t+1}\right) \stackrel{\text { def }}{=} p\left(x, r^{1} \oplus \ldots \oplus r^{t+1}\right)$ (where $t$ is a bound on the number of corrupted parties), assign each input vector $r^{j}$ to a distinct player, and instruct it to pick it at random. (See Ishai \& Kushilevitz 2000 for more details.) This second reduction step is also non-interactive. Thus, any secure protocol for $\hat{f}^{\prime}$ or $\hat{f}$ gives rise to a secure protocol for $f$ with the same number of rounds. The non-interactive nature of the reduction makes it insensitive to almost all aspects of the security model.

Previous constructions of (perfect or statistical) randomizing polynomials (Cramer et al. 2003; Ishai \& Kushilevitz 2000, 2002) provided informationtheoretic reductions of the type discussed above. In particular, if the protocol used for evaluating $\hat{f}^{\prime}$ is information-theoretically secure, then so is the resulting protocol for $f$. The main limitation of these previous reductions is that they efficiently apply only to restricted classes of functions, typically related to different log-space classes. This situation is remedied in the current work, where we obtain (under the EPRG assumption) a general secure reduction from a function $f$ to a related degree-3 function $\hat{f}^{\prime}$. The main price we pay is that the security of the reduction is no longer information-theoretic. Thus, even if the underlying protocol for $\hat{f}^{\prime}$ is secure in the information-theoretic sense, the resulting protocol for $f$ will only be computationally secure.

To formulate the above we need the following definitions.

Definition 5.8 (Secure computation). Let $f\left(x_{1}, \ldots, x_{n}\right)$ be an $m$-party functionality, i.e., a (possibly randomized) mapping from $m$ inputs of equal length into $m$ outputs. Let $\pi$ be an $m$-party protocol. We formulate the require- 
ment that $\pi$ securely computes $f$ by comparing the following "real process" and "ideal process".

The real process. A $t$-bounded adversary $A$ attacking the real process is a probabilistic polynomial-time algorithm, who may corrupt up to $t$ parties and observe all of their internal data. At the end of the interaction, the adversary may output an arbitrary function of its view, which consists of the inputs, the random coin tosses, and the incoming messages of the corrupted parties. We distinguish between passive vs. active adversaries and between adaptive vs. non-adaptive adversaries. If the adversary is active, it has full control over the messages sent by the corrupted parties, whereas if it is passive, it follows the protocol's instructions (but may try to deduce information by performing computations on observed data). When the set of corrupted parties has to be chosen in advance, we say that the adversary is non-adaptive, and otherwise say that it is adaptive. Given an $m$-tuple of inputs $\left(x_{1}, \ldots, x_{m}\right) \in\left(\{0,1\}^{n}\right)^{m}$, the output of the real process is defined as the random variable containing the concatenation of the adversary's output with the outputs and identities of the uncorrupted parties. We denote this output by $\operatorname{REAL}_{\pi, A}\left(x_{1}, \ldots, x_{m}\right)$.

The ideal process. In the ideal process, an incorruptible trusted party is employed for computing the given functionality. That is, the "protocol" in the ideal process instructs each party to send its input to the trusted party, who computes the functionality $f$ and sends to each party its output. The interaction of a $t$-bounded adversary $A^{\prime}$ with the ideal process and the output of the ideal process are defined analogously to the above definitions for the real process. The adversary attacking the ideal process will also be referred to as a simulator. We denote the output of the ideal process on the inputs $\left(x_{1}, \ldots, x_{m}\right) \in\left(\{0,1\}^{n}\right)^{m}$ by $\operatorname{IdEAL}_{f, A^{\prime}}\left(x_{1}, \ldots, x_{m}\right)$.

The protocol $\pi$ is said to $t$-securely realize the given functionality $f$ with respect to a specified type of adversary (namely, passive or active, adaptive or non-adaptive) if for any probabilistic polynomial-time $t$-bounded adversary $A$ attacking the real process, there exists a probabilistic polynomialtime $t$-bounded simulator $A^{\prime}$ attacking the ideal process, such that for any sequence $\left\{\bar{x}_{n}\right\}$ of $m$-tuples such that $\bar{x}_{n} \in\left(\{0,1\}^{n}\right)^{m}$, we have $\operatorname{REAL}_{\pi, A}\left(\bar{x}_{n}\right) \stackrel{c}{=}$ $\operatorname{IDEAL}_{f, S}\left(\bar{x}_{n}\right)$.

Secure reductions. To define secure reductions, consider the following hybrid model. An $m$-party protocol augmented with an oracle to the $m$-party 
functionality $g$ is a standard protocol in which the parties are allowed to invoke $g$, i.e., a trusted party to which they can securely send inputs and receive the corresponding outputs. The notion of $t$-security generalizes to protocols augmented with an oracle in the natural way.

Definition 5.9. Let $f$ and $g$ be $m$-party functionalities. A $t$-secure reduction from $f$ to $g$ is an $m$-party protocol that given an oracle access to the functionality $g, t$-securely realizes the functionality $f$ (with respect to a specified type of adversary). We say that the reduction is non-interactive if it involves a single call to $f$ (and possibly local computations on inputs and outputs), but no further communication.

Appropriate composition theorems (e.g. Theorems 7.3.3 and 7.4.3 in Goldreich 2004) guarantee that the call to $g$ can be replaced by any secure protocol realizing $g$, without violating the security of the high-level protocol for $f .{ }^{5}$ In the above terminology, Theorem 4.14 has the following corollary.

Theorem 5.10. Suppose the EPRG assumption holds. Let $f\left(x_{1}, \ldots, x_{m}\right)$ be an $m$-party functionality computed by a (uniform) circuit family of size $s(n)$. Then, for any $\epsilon>0$, there is a non-interactive, computationally $(m-1)$-secure reduction from $f$ to either of the following two efficient functionalities:

- A randomized functionality $\hat{f}\left(x_{1}, \ldots, x_{m}\right)$ of degree 3 (over $\mathrm{GF}(2)$ ) with a random input and output of length $O\left(s(n) \cdot n^{\epsilon}\right)$ each;

- A deterministic functionality $\hat{f}^{\prime}\left(x_{1}^{\prime}, \ldots, x_{m}^{\prime}\right)$ of degree 3 (over $\left.\mathrm{GF}(2)\right)$ with input length $O\left(m \cdot s(n) \cdot n^{\epsilon}\right)$ and output length $O\left(s(n) \cdot n^{\epsilon}\right)$.

Both reductions are non-interactive in the sense that they involve a single call to $\hat{f}$ or $\hat{f}^{\prime}$ and no further interaction. They both apply regardless of whether the adversary is passive or active, adaptive or non-adaptive.

Proof. The second item follows from the first via standard (non-interactive, degree-preserving) secure reduction from randomized functionalities to deterministic functionalities (see Goldreich 2004, Prop. 7.3.4). Thus we will only

\footnotetext{
${ }^{5}$ Actually, for the composition theorem to go through, Definition 5.8 should be augmented by providing players and adversaries with auxiliary inputs. We ignore this technicality here, and note that the results in this section apply (with essentially the same proofs) to the augmented model as well.
} 
prove the first item. Assume, without loss of generality, that $f$ is a deterministic functionality that returns the same output to all the parties. ${ }^{6}$ Let $\hat{f}(x, r)$ be the computational encoding of $f(x)$ promised by Theorem 4.14. (Recall that $\hat{f}$ is indeed a degree-3 function having $O\left(s(n) \cdot n^{\epsilon}\right)$ random inputs and outputs.) The following protocol $(m-1)$-securely reduces the computation of $f(x)$ to $\hat{f}(x, r)$ (where $\hat{f}$ is viewed as a randomized functionality whose randomness is $r$ ).

○ Inputs: Party $i$ gets input $x_{i} \in\{0,1\}^{n}$.

- Party $i$ invokes the (randomized) oracle $\hat{f}$ with query $x_{i}$, and receives an output $\hat{y}$.

- Outputs: Each party locally applies the decoder $B$ of the encoding to the answer $\hat{y}$ received from the oracle, and outputs the result.

We start by showing that the reduction is $(m-1)$-secure against a passive non-adaptive adversary. Let $A$ be such an adversary that attacks some set $I \subset[m]$ of the players. Then the output of the real process is

$$
\left(A\left(x_{I}, \hat{f}(x, r)\right), B(\hat{f}(x, r)), \bar{I}\right)
$$

where $x_{I}=\left(x_{i}\right)_{i \in I}, \bar{I} \stackrel{\text { def }}{=}[m] \backslash I$ and $r$ is a uniformly chosen string of an appropriate length. We define a (passive non-adaptive) simulator $A^{\prime}$ that attacks the ideal process in the natural way: that is, $A^{\prime}\left(x_{I}, y\right)=A\left(x_{I}, S(y)\right)$, where $y$ is the answer received from the trusted party (i.e., $f(x))$ and $S$ is the computationally private simulator of the encoding. Thus, the output of the ideal process is $\left(A^{\prime}\left(x_{I}, f(x)\right), f(x), \bar{I}\right)$. By the definition of $A^{\prime}$, the privacy of the encoding $\hat{f}$ and Fact 2.3, we have

$$
\begin{aligned}
\operatorname{IDEAL}(x) & \equiv\left(A\left(x_{I}, S(f(x))\right), f(x), \bar{I}\right) \stackrel{c}{\equiv}\left(A\left(x_{I}, \hat{f}(x, r)\right), B(\hat{f}(x, r)), \bar{I}\right) \\
& \equiv \operatorname{REAL}(x),
\end{aligned}
$$

which finishes the proof.

We now sketch the security proof for the case of an adversary $A$ which is both adaptive and active. (The non-adaptive active case as well as the adaptive passive case are treated similarly.) An attack by $A$ has the following form:

\footnotetext{
${ }^{6}$ To handle randomized functionalities we use the non-interactive secure reduction mentioned above. Now, we can $(m-1)$-securely reduce $f$ to a single-output functionality by letting each party to mask its output $f_{i}$ with a private randomness. That is, $f^{\prime}\left(\left(x_{1}, r_{1}\right) \ldots,\left(x_{m}, r_{m}\right)\right)=\left(\left(f_{1}\left(x_{1}\right) \oplus r_{1}\right) \circ \cdots \circ\left(f_{1}\left(x_{m}\right) \oplus r_{m}\right)\right)$. As both reductions are non-interactive the resulting reduction is also non-interactive. Moreover, the circuit size of $f^{\prime}$ is linear in the size of the circuit that computes the original function.
} 
(1) Before calling the oracle $\hat{f}$, in each step $A$ may decide (according to his current view) to corrupt some party $i$ and learn its input $x_{i}$. (2) When the oracle $\hat{f}$ is invoked $A$ changes the input of each corrupted party $i$ to some value $x_{i}^{\prime}$, which is handed to the $\hat{f}$ oracle. (3) After the parties call the oracle on some (partially corrupted) input $x^{\prime}=\left(x_{I}^{\prime}, x_{\bar{I}}\right)$, the oracle returns a randomized encoding $\hat{f}\left(x^{\prime}\right)$ to the adversary, and now $A$ may adaptively corrupt additional parties. Finally, $A$ outputs some function of its entire view. For every such adversary we construct a simulator $A^{\prime}$ that attacks the ideal process by invoking $A$ and emulating his interaction with the real process. Namely, (1) before the call to the trusted party we let $A$ choose (in each step) which party to corrupt and feed it with the input we learn; (2) when the trusted party is invoked we let $A$ pick $x_{I}^{\prime}$ according to its current view and send these $x_{I}^{\prime}$ to the $f$ oracle; (3) given the result $y=f\left(x_{I}^{\prime}, x_{\bar{I}}\right)$ returned by the oracle, we invoke the simulator (of the encoding) on $y$ and feed the result to $A$. Finally, we let $A$ pick new corrupted players as in step (1). We claim that in each step of the protocol the view of $A$ when interacting with the real process is computationally indistinguishable from the view of $A$ when it is invoked by $A^{\prime}$ in the ideal process. (In fact, before the call to the oracle these views are identically distributed.) Hence, the outputs of the two processes are also computationally indistinguishable.

A high-level corollary of Theorem 5.10 is that computing arbitrary polynomial-time computable functionalities is as easy as computing degree-3 functionalities. Thus, when designing new MPC protocols, it suffices to consider degree-3 functionalities which are often easier to handle.

More concretely, Theorem 5.10 gives rise to new, conceptually simpler, constant-round protocols for general functionalities. For instance, a combination of this result with the "BGW protocol" (Ben-Or et al. 1988) gives a simpler alternative to the constant-round protocol of Beaver et al. (1990). The resulting protocol will be more round-efficient, and in some cases (depending on the number of parties and the "easiness" of the PRG) even more communicationefficient than the protocol of Beaver et al. (1990). On the downside, Theorem 5.10 relies on a stronger assumption than the protocol from Beaver et al. (1990) (an easy PRG vs. an arbitrary PRG).

An interesting open question, which is motivated mainly from the point of view of the MPC application, is to come up with an "arithmetic" variant of the construction. That is, given an arithmetic circuit $C$, say with addition and multiplication gates, construct a vector of computationally private randomizing polynomials of size poly $(|C|)$ which makes a black-box use of the underlying field. The latter requirement means that the same polynomials should represent 
$C$ over any field, ruling out the option of simulating arithmetic field operations by boolean operations. Such a result is known for weaker arithmetic models such as formulas and branching programs (see Cramer et al. 2003).

\section{Proof of Lemma 4.10}

The simulator. We start with the description of the simulator $S$. Given $1^{n}$ and $f_{n}(x)$, for some $x \in\{0,1\}^{n}$, the simulator chooses, for every wire $i$ of the circuit $C_{n}$, an active key and a color; namely, $S$ selects a random string $W_{i}^{b_{i}}$ of length $2 k(n)$, and a random bit $c_{i}$. (Recall that $b_{i}$ denotes the value of the $i$-th wire induced by the input $x$. The simulator, of course, does not know this value.) For an input wire $i$, the simulator outputs $W_{i}^{b_{i}} \circ c_{i}$. For a gate $t$ with input wires $i, j$ and output wires $y_{1}, \ldots, y_{m}$ the simulator computes the active label $Q_{t}^{c_{i}, c_{j}}=E_{W_{i}^{b_{i}, c_{j}} \oplus W_{j}^{b_{j}, c_{i}}}\left(W_{y_{1}}^{b_{y_{1}}} \circ c_{y_{1}} \circ \cdots \circ W_{y_{m}}^{b_{y_{m}}} \circ c_{y_{m}}\right)$ and sets the other three inactive labels of this gate to be encryptions of all-zeros strings of appropriate length under random keys; that is, for any two bits $\left(a_{i}, a_{j}\right) \neq\left(c_{i}, c_{j}\right)$, the simulator chooses uniformly a $k(n)$-bit string $R_{a_{i}, a_{j}}$ and

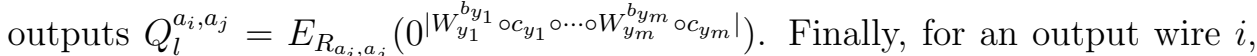
the simulator outputs $r_{i}=c_{i}-b_{i}$ (recall that $b_{i}$ is known since $f_{n}(x)$ is given).

Since $C_{n}$ can be constructed in polynomial time and since the encryption algorithm runs in polynomial time the simulator is also a polynomial-time algorithm. We refer to the gate labels constructed by the simulator as "fake" gate labels and to gate labels of $\hat{f}_{n}$ as "real" gate labels.

Assume, towards a contradiction, that there exists a (non-uniform) polynomial-size circuit family $\left\{A_{n}\right\}$, a polynomial $p(\cdot)$, and a string family $\left\{x_{n}\right\}$ with $\left|x_{n}\right|=n$ such that for infinitely many $n$ 's,

$$
\Delta(n) \stackrel{\text { def }}{=}\left|\operatorname{Pr}\left[A_{n}\left(S\left(1^{n}, f_{n}\left(x_{n}\right)\right)\right)=1\right]-\operatorname{Pr}\left[A_{n}\left(\hat{f}_{n}\left(x_{n}, U_{\mu(n)}\right)\right)=1\right]\right|>\frac{1}{p(n)} .
$$

We use a hybrid argument to show that such a distinguisher can be used to break the encryption, hence deriving a contradiction.

From now on we fix $n$ and let $k=k(n)$. We construct a sequence of hybrid distributions that depend on $x_{n}$, and mix "real" gates labels and "fake" ones, so that one endpoint corresponds to the simulated output (in which all the gates have "fake" labels) and the other endpoint corresponds to $\hat{f}_{n}\left(x_{n}, U_{\mu(n)}\right)$ (in which all the gates have real labels). Hence, if the extreme hybrids can be efficiently distinguished then there must be two neighboring hybrids that can be efficiently distinguished. 
The hybrids $H_{t}^{n}$. First, we order the gates of $C_{n}$ in topological order. That is, if the gate $t$ uses the output of gate $t^{\prime}$, then $t^{\prime}<t$. Now, for every $t=$ $0, \ldots, \Gamma(n)$, we define the hybrid algorithm $H_{t}^{n}$ that constructs "fake" labels for the first $t$ gates and "real" labels for the rest of the gates:

1. For every wire $i$ uniformly choose two $2 k$-bit strings $W_{i}^{b_{i}}, W_{i}^{1-b_{i}}$ and a random bit $c_{i}$.

2. For every input wire $i$ output $W_{i}^{b_{i}} \circ c_{i}$.

3. For every gate $t^{\prime} \leq t$ with input wires $i, j$ and output wires $y_{1}, \ldots, y_{m}$ output

$$
Q_{t^{\prime}}^{c_{i}, c_{j}}=E_{W_{i}^{b_{i}, c_{j}} \oplus W_{j}^{b_{j}, c_{i}}}\left(W_{y_{1}}^{b_{y_{1}}} \circ c_{y_{1}} \circ \cdots \circ W_{y_{m}}^{b_{y_{m}}} \circ c_{y_{m}}\right),
$$

and for every choice of $\left(a_{i}, a_{j}\right) \in\{0,1\}^{2}$ that is different from $\left(c_{i}, c_{j}\right)$, uniformly choose a $k$-bit string $R_{a_{i}, a_{j}}$ and output

$$
Q_{t^{\prime}}^{a_{i}, a_{j}}=E_{R_{a_{i}, a_{j}}}\left(0^{\left|W_{y_{1}}^{b_{y_{1}}} \circ c_{y_{1}} \circ \cdots \circ W_{y m}^{b_{y_{m}}} \circ c_{y_{m}}\right|}\right)
$$

4. For every gate $t^{\prime}>t$, let $g$ be the function that $t^{\prime}$ computes (AND or OR), let $i, j$ be the input wires of $t^{\prime}$ and let $y_{1}, \ldots, y_{m}$ be its output wires. Use $x_{n}$ to compute the value of $b_{i}\left(x_{n}\right), b_{j}\left(x_{n}\right)$, and set $r_{i}=c_{i}-b_{i}$ and $r_{j}=c_{j}-b_{j}$. For every choice of $\left(a_{i}, a_{j}\right) \in\{0,1\}^{2}$, compute $Q_{t^{\prime}}^{a_{i}, a_{j}}$ exactly as in Construction 4.7, and output it.

5. For every output wire $i$ compute $b_{i}$ and output $r_{i}=c_{i}-b_{i}$.

Claim 6.1. There exist some $0 \leq t \leq \Gamma(n)-1$ such that $A_{n}$ distinguishes between $H_{t}^{n}$ and $H_{t+1}^{n}$ with advantage $\Delta(n) / \Gamma(n)$.

Proof. First, note that $H_{t}^{n}$ uses the string $x_{n}$ only when constructing real labels, that is, in Step 4. Steps 1-3 can be performed without any knowledge on $x_{n}$, and Step 5 requires only the knowledge of $f_{n}\left(x_{n}\right)$. Obviously, the algorithm $H_{\Gamma(n)}^{n}$ is just a different description of the simulator $S$, and therefore $S\left(1^{n}, f_{n}\left(x_{n}\right)\right) \equiv H_{\Gamma(n)}^{n}$. We also claim that the second extreme hybrid, $H_{0}^{n}$, coincides with the distribution of the "real" encoding, $\hat{f}_{n}\left(x_{n}, U_{\mu(n)}\right)$. To see this note that (1) the strings $W_{i}^{0}, W_{i}^{1}$ are chosen uniformly and independently by $H_{0}^{n}$, as they are in $\hat{f}_{n}\left(x_{n}, U_{\mu(n)}\right)$; and (2) since $H_{0}^{n}$ chooses the $c_{i}$ 's uniformly and independently and sets $r_{i}=c_{i}-b_{i}$ it follows that the $r_{i}$ 's themselves are also 
distributed uniformly and independently exactly as they are in $\hat{f}_{n}\left(x_{n}, U_{\mu(n)}\right)$. Since for every gate $t$ the value of $Q_{t}^{a_{i}, a_{j}}$ is a function of the random variables, and since it is computed by $H_{0}^{n}$ in the same way as in $\hat{f}_{n}\left(x_{n}, U_{\mu(n)}\right)$, we conclude that $H_{0}^{n} \equiv \hat{f}_{n}\left(x_{n}, U_{\mu(n)}\right)$.

Hence, we can write

$$
\begin{aligned}
\Delta(n) & =\left|\operatorname{Pr}\left[A_{n}\left(H_{0}^{n}\right)=1\right]-\operatorname{Pr}\left[A_{n}\left(H_{\Gamma(n)}^{n}\right)=1\right]\right| \\
& \leq \sum_{t=0}^{\Gamma(n)-1}\left|\operatorname{Pr}\left[A_{n}\left(H_{t}^{n}\right)=1\right]-\operatorname{Pr}\left[A_{n}\left(H_{t+1}^{n}\right)=1\right]\right|,
\end{aligned}
$$

and so there exists some $0 \leq t \leq \Gamma(n)-1$ such that $A_{n}$ distinguishes between $H_{t}^{n}$ and $H_{t+1}^{n}$ with advantage $\Delta(n) / \Gamma(n)$.

We now show that distinguishing $H_{t}^{n}$ and $H_{t+1}^{n}$ allows one to distinguish whether a single gate is real or fake. To do this, we define two random experiments $P_{n}(0)$ and $P_{n}(1)$ that produce a real gate and a fake gate, respectively.

The experiments $P_{n}(0), P_{n}(1)$. Let $i, j$ be the input wires of the gate $t$, let $y_{1}, \ldots, y_{m}$ be the output wires of $t$, let $g$ be the function that gate $t$ computes, and let $b_{i}, b_{j}, b_{y_{1}}, \ldots, b_{y_{m}}$ be the values of the corresponding wires induced by the input $x_{n}$. For $\sigma \in\{0,1\}$, define the distribution $P_{n}(\sigma)$ as the output distribution of the following random process:

○ Uniformly choose the following $2 k$-bit strings: $W_{i}^{b_{i}}, W_{i}^{1-b_{i}}, W_{j}^{b_{j}}, W_{j}^{1-b_{j}}$, $W_{y_{1}}^{b_{y_{1}}}, W_{y_{1}}^{1-b_{y_{1}}}, \ldots, W_{y_{m}}^{b_{y_{m}}}, W_{y_{m}}^{1-b_{y_{m}}}$, and the random bits $c_{i}, c_{j}, c_{y_{1}}, \ldots, c_{y_{m}}$.

○ If $\sigma=0$ then set $Q_{t}^{c_{i}, c_{j}}$ and the other three $Q_{t}^{a_{i}, a_{j}}$ exactly as in Step 3 of $H_{t}^{n}$.

○ If $\sigma=1$ then set $Q_{t}^{a_{i}, a_{j}}$ exactly as in Step 4 of $H_{t}^{n}$; that is, set $r_{i}=c_{i}-b_{i}$, $r_{j}=c_{j}-b_{j}$, and for every choice of $\left(a_{i}, a_{j}\right) \in\{0,1\}^{2}$, let $Q_{t}^{a_{i}, a_{j}}$ be

$$
\begin{aligned}
E_{W_{i}^{a_{i}-r_{i}, a_{j}} \oplus W_{j}^{a_{j}-r_{j}, a_{i}}}\left(W_{y_{1}}^{g\left(a_{i}-r_{i}, a_{j}-r_{j}\right)} \circ\left(g\left(a_{i}-r_{i}, a_{j}-r_{j}\right)+r_{y_{1}}\right) \circ \cdots\right. \\
\left.\circ W_{y_{m}}^{g\left(a_{i}-r_{i}, a_{j}-r_{j}\right)} \circ\left(g\left(a_{i}-r_{i}, a_{j}-r_{j}\right)+r_{y_{m}}\right)\right) .
\end{aligned}
$$

○ Output $\left(W_{i}^{b_{i}}, W_{j}^{b_{j}}, W_{y_{1}}^{b_{y_{1}}}, W_{y_{1}}^{1-b_{y_{1}}}, \ldots, W_{y_{m}}^{b_{y_{m}}}, W_{y_{m}}^{1-b_{y_{m}}}, c_{i}, c_{j}, c_{y_{1}}, \ldots, c_{y_{m}}\right.$, $\left.Q_{t}^{0,0}, Q_{t}^{0,1}, Q_{t}^{1,0}, Q_{t}^{1,1}\right)$. 
Claim 6.2. There exists a polynomial size circuit $A_{n}^{\prime}$ that distinguishes between $P_{n}(0)$ and $P_{n}(1)$ with advantage $\Delta(n) / \Gamma(n)$.

Proof. The adversary $A_{n}^{\prime}$ uses the output of $P_{n}(\sigma)$ to construct one of the hybrids $H_{t}^{n}$ and $H_{t+1}^{n}$, and then uses $A_{n}$ to distinguish between them. Namely, given the output of $P_{n}$, the distinguisher $A_{n}^{\prime}$ invokes the algorithm $H_{t}^{n}$ where the values of the strings $\left(W_{i}^{b_{i}}, W_{j}^{b_{j}}, W_{y_{1}}^{b_{y_{1}}}, W_{y_{1}}^{1-b_{y_{1}}}, \ldots, W_{y_{m}}^{b_{y_{m}}}, W_{y_{m}}^{1-b_{y_{m}}}, c_{i}, c_{j}, c_{y_{1}}, \ldots\right.$, $\left.c_{y_{m}}, Q_{t}^{0,0}, Q_{t}^{0,1}, Q_{t}^{1,0}, Q_{t}^{1,1}\right)$ are set to the values given by $P_{n}$. By the definition of $P_{n}$, when $P_{n}(0)$ is invoked we get the distribution of $H_{t}^{n}$, that is, the gate $t$ is "fake"; on the other hand, if $P_{n}(1)$ is invoked then the gate $t$ is "real" and we get the distribution of $H_{t+1}^{n}$. Hence, by Claim 6.1, $A_{n}^{\prime}$ has the desired advantage. Finally, since $H_{t}^{n}$ runs in polynomial time (when $x_{n}$ is given), the size of $A_{n}^{\prime}$ is indeed polynomial.

A delicate point. Note that $P_{n}$ does not output the inactive keys of the wires $i$ and $j$ (which is crucial for Claim 6.3 to hold). However, the hybrid distributions use inactive keys of wires that either enter a real gate or leave a real gate (in the first case the inactive keys are used as the keys of the gate label encryption, whereas in the latter case, the inactive keys are being encrypted). Hence, we do not need these inactive keys to construct the rest of the distribution $H_{t}^{n}$ (or $H_{t+1}^{n}$ ), as $i$ and $j$ are output wires of gates that precede $t$ and therefore are "fake" gates. This is the reason for which we had to sort the gates. On the other hand, the process $P_{n}$ must output the inactive keys of the output wires of the gate $y_{1}, \ldots, y_{m}$, since these wires might enter as inputs to another gate $t^{\prime}>t$ which is a "real" gate in both $H_{t}^{n}$ and $H_{t+1}^{n}$.

We now define a related experiment $P_{n}^{\prime}$ in which some of the randomness used by $P_{n}$ is fixed. Specifically, we fix the random strings $W_{y_{1}}^{b_{y_{1}}}, W_{y_{1}}^{1-b_{y_{1}}}, \ldots$, $W_{y_{m}}^{b_{y_{m}}}, W_{y_{m}}^{1-b_{y_{m}}}, c_{i}, c_{j}, c_{y_{1}}, \ldots, c_{y_{m}}$ to some value such that the resulting experiments can still be distinguished by $A_{n}^{\prime}$ with advantage $\Delta(n) / \Gamma(n)$. (Existence of such strings is promised by an averaging argument.) For simplicity, we omit the fixed strings from the output of this new experiment. The experiments $P_{n}^{\prime}(0)$ and $P_{n}^{\prime}(1)$ can still be distinguished by some polynomial-size circuit with advantage $\Delta(n) / \Gamma(n)$. (Such distinguisher can be constructed by incorporating the omitted fixed strings into $A_{n}^{\prime}$.) Hence, by the contradiction hypothesis, it follows that this advantage is greater than $1 / \Gamma(n) p(n)$ for infinitely many $n$ 's. As $\Gamma(n)$ is polynomial in $n$ (since $C_{n}$ is of polynomial size) we deduce that the distribution ensembles $\left\{P_{n}^{\prime}(0)\right\}_{n \in \mathbb{N}}$ and $\left\{P_{n}^{\prime}(1)\right\}_{n \in \mathbb{N}}$ are not computationally indistinguishable, in contradiction with the following claim. 
Claim 6.3. $\left\{P_{n}^{\prime}(0)\right\}_{n \in \mathbb{N}} \stackrel{c}{\equiv}\left\{P_{n}^{\prime}(1)\right\}_{n \in \mathbb{N}}$.

Proof. Fix some $n$. For both distributions $P_{n}^{\prime}(0)$ and $P_{n}^{\prime}(1)$, the first two entries (i.e., $W_{i}^{b_{i}}, W_{j}^{b_{j}}$ ) are two uniformly and independently chosen $2 k(n)$-length strings, and the active label $Q_{t}^{b_{i}, b_{j}}$ is a function of $W_{i}^{b_{i}}, W_{j}^{b_{j}}$ and the fixed strings. Hence, the distributions $P_{n}^{\prime}(0)$ and $P_{n}^{\prime}(1)$ differ only in the inactive labels $Q_{t}^{a_{i}, a_{j}}$ for $\left(a_{i}, a_{j}\right) \neq\left(c_{i}, c_{j}\right)$. In $P_{n}^{\prime}(0)$ each of these entries is an all-zeros string that was encrypted under uniformly and independently chosen key $R_{a_{i}, a_{j}}$. In the second distribution $P_{n}^{\prime}(1)$, the entry $Q_{t}^{a_{i}, a_{j}}$ is an encryption of a "meaningful" message that was encrypted under the key $W_{i}^{a_{i}-r_{i}, a_{j}} \oplus W_{j}^{a_{j}-r_{j}, a_{i}}$. Since $\left(a_{i}, a_{j}\right) \neq\left(c_{i}, c_{j}\right)$ at least one of the strings $W_{i}^{a_{i}-r_{i}, a_{j}}, W_{j}^{a_{j}-r_{j}, a_{i}}$ is not given in the output of $P_{n}^{\prime}(1)$ as part of $W_{i}^{b_{i}}, W_{j}^{b_{j}}$. Also, each of the strings $W_{i}^{a_{i}-r_{i}, a_{j}}, W_{j}^{a_{j}-r_{j}, a_{i}}$ was chosen uniformly and it appears only in $Q_{t}^{a_{i}, a_{j}}$ and not in any of the other gate labels, therefore the key $W_{i}^{a_{i}-r_{i}, a_{j}} \oplus W_{j}^{a_{j}-r_{j}, a_{i}}$ is distributed uniformly and independently of the other entries of $P_{n}^{\prime}(1)$ 's output. So all the entries of both $P_{n}^{\prime}(1)$ and $P_{n}^{\prime}(0)$ are independent. Moreover, the security of the encryption scheme implies that the ensemble $\left\{Q_{t}^{a_{i}, a_{j}}\right\}$ for $\left(a_{i}, a_{j}\right) \neq\left(c_{i}, c_{j}\right)$ produced by $P_{n}^{\prime}(1)$ is computationally indistinguishable from the corresponding ensemble produced by $P_{n}^{\prime}(0)$, as in both cases some $p(n)$-length message is encrypted under uniformly chosen $k(n)$-length key. (Recall that $k(n)$ is polynomial in $n$ by definition, and $\left.p(n)=O\left(\left|C_{n}\right| k(n)\right)=\operatorname{poly}(n)\right)$. Hence, by Fact 2.2 , the claim follows.

\section{Acknowledgements}

We thank Omer Reingold for helpful discussions. Research supported by grant no. 36/03 from the Israel Science Foundation.

\section{References}

B. Applebaum, Y. Ishai \& E. Kushilevitz (2004). Cryptography in $\mathrm{NC}^{0}$. In Proc. 45th FOCS, 166-175. Full version to appear in SICOMP, Special Issue on Randomness in Computation.

D. Beaver, S. Micali \& P. Rogaway (1990). The round complexity of secure protocols (extended abstract). In Proc. 22nd STOC, 503-513.

M. Ben-Or, S. Goldwasser \& A. Wigderson (1988). Completeness theorems for non-cryptographic fault-tolerant distributed computation. In Proc. 20th STOC, 1-10. 
M. Blum (1983). Coin flipping by telephone: a protocol for solving impossible problems. SIGACT News 15, 23-27.

M. Blum \& S. MiCALI (1984). How to generate cryptographically strong sequences of pseudo-random bits. SIAM J. Comput. 13, 850-864. Preliminary version in Proc. 23rd FOCS, 1982.

R. CANETti (2000). Security and composition of multiparty cryptographic protocols. J. Cryptology 13, 143-202.

R. Canetti (2001). Universally composable security: a new paradigm for cryptographic protocols. In Proc. 42nd FOCS, 136-145.

D. Chaum, C. Crépeau \& I. Damgård (1988). Multiparty unconditionally secure protocols (extended abstract). In Proc. 20th STOC, 11-19.

R. Cramer, S. Fehr, Y. Ishai \& E. Kushilevitz (2003). Efficient multi-party computation over rings. In Proc. EUROCRYPT '03, 596-613.

J. Feigenbaum (1993). Locally random reductions in interactive complexity theory. In Advances in Computational Complexity Theory, DIMACS Ser. Discrete Math. Theoret. Comput. Sci. 13, 73-98.

O. Goldreich (2001). Foundations of Cryptography: Basic Tools. Cambridge Univ. Press.

O. Goldreich (2004). Foundations of Cryptography: Basic Applications. Cambridge Univ. Press.

O. Goldreich, S. Goldwasser \& S. Micali (1986). How to construct random functions. J. ACM 33, 792-807.

O. Goldreich \& L. A. Levin (1989). A hard-core predicate for all one-way functions. In Proc. 21st STOC, 25-32.

O. Goldreich, S. Micali \& A. Wigderson (1987). How to play any mental game (extended abstract). In Proc. 19th STOC, 218-229.

S. Goldwasser \& S. Micali (1984). Probabilistic encryption. J. Comput. System Sci. 28, 270-299. Preliminary version in Proc. 14th STOC, 1982.

I. Haitner, D. Harnik \& O. Reingold (2005). On the power of the randomized iterate. In Proc. Crypto 2006 (to appear). Also appeared in ECCC - TR05-135.

J. Håstad, R. Impagliazzo, L. A. Levin \& M. Luby (1999). A pseudorandom generator from any one-way function. SIAM J. Comput. 28, 1364-1396. 
Y. Ishai \& E. Kushilevitz (2000). Randomizing polynomials: a new representation with applications to round-efficient secure computation. In Proc. 41st FOCS, 294304.

Y. Ishai \& E. Kushilevitz (2002). Perfect constant-round secure computation via perfect randomizing polynomials. In Proc. 29th ICALP, 244-256.

Y. Lindell \& B. Pinkas (2004). A proof of Yao's protocol for secure two-party Computation. Electronic Colloquium on Computational Complexity 11(063).

N. Linial, Y. Mansour \& N. Nisan (1993). Constant depth circuits, Fourier transform, and learnability. J. ACM 40,607-620. Preliminary version in Proc. 30th FOCS, 1989.

M. NAOR (1991). Bit commitment using pseudorandomness. J. Cryptology 4, 151158.

M. NaOr, B. Pinkas \& R. Sumner (1999). Privacy preserving auctions and mechanism design. In Proc. 1st ACM Conference on Electronic Commerce, 129-139.

M. NAOR \& O. Reingold (1999). Synthesizers and their application to the parallel construction of pseudo-random functions. J. Comput. System Sci. 58, 336-375. Preliminary version in Proc. 36th FOCS, 1995.

M. NAOR \& O. ReingOld (2004). Number-theoretic constructions of efficient pseudo-random functions. J. ACM 51, 231-262. Preliminary version in Proc. 38th FOCS, 1997.

O. Reingold, L. Trevisan \& S. Vadhan (2004). Notions of reducibility between cryptographic primitives. In Theory of Cryptography, Lecture Notes in Comput. Sci. 2951, 1-20.

P. Rogaway (1991). The round complexity of secure protocols. Ph.D. thesis, MIT.

C. E. Shannon (1949). Communication theory of secrecy systems. Bell System Technical J. 28-4, 656-715.

S. R. TATE \& K. XU (2003). On garbled circuits and constant round secure function evaluation. CoPS Lab Technical Report 2003-02, Univ. of North Texas.

E. Viola (2005). On constructing parallel pseudorandom generators from one-way functions. In Proc. 20th Conference on Computational Complexity (CCC), 183-197.

A. Wigderson (1994). NL/poly $\subseteq \oplus \mathrm{L} /$ poly. In Proc. 9th Structure in Complexity Theory Conference, 59-62. 
A. C. YaO (1982). Theory and application of trapdoor functions. In Proc. 23rd FOCS, 80-91.

A. C. YAo (1986). How to generate and exchange secrets. In Proc. 27th FOCS, $162-167$.

Manuscript received 16 September 2005

\author{
Benny Applebaum \\ Computer Science Department \\ Haifa 32000, Israel \\ abenny@cs.technion.ac.il \\ EyAl Kushilevitz \\ Computer Science Department \\ Technion - Israel Institute of Technology \\ Haifa 32000, Israel \\ eyalk@cs.technion.ac.il
}

YUVAL ISHAI

Computer Science Department

Technion - Israel Institute of Technology Technion - Israel Institute of Technology

Haifa 32000, Israel

yuvali@cs.technion.ac.il 